ARTICLE

https://doi.org/10.1038/s41467-019-10496-3

\title{
Cryo-electron microscopy structure of an archaeal ribonuclease P holoenzyme
}

Futang Wan 1,2,3,8, Qianmin Wang 4,5,8, Jing Tan ${ }^{1,2,8}$, Ming Tan (1) ${ }^{1,2}$, Juan Chen ${ }^{4,5}$, Shaohua Shi ${ }^{4,5}$, Pengfei Lan (1) 4,5, Jian Wu ${ }^{4,5,6}$ \& Ming Lei (1) 4,5,7

Ribonuclease $\mathrm{P}$ (RNase $\mathrm{P}$ ) is an essential ribozyme responsible for tRNA $5^{\prime}$ maturation. Here we report the cryo-EM structures of Methanocaldococcus jannaschii (Mja) RNase P holoenzyme alone and in complex with a tRNA substrate at resolutions of $4.6 \AA$ and $4.3 \AA$, respectively. The structures reveal that the subunits of MjaRNase $\mathrm{P}$ are strung together to organize the holoenzyme in a dimeric conformation required for efficient catalysis. The structures also show that archaeal RNase $P$ is a functional chimera of bacterial and eukaryal RNase Ps that possesses bacterial-like two RNA-based anchors and a eukaryal-like proteinaided stabilization mechanism. The $3^{\prime}$-RCCA sequence of tRNA, which is a key recognition element for bacterial RNase $\mathrm{P}$, is dispensable for tRNA recognition by MjaRNase $\mathrm{P}$. The overall organization of MjaRNase $\mathrm{P}$, particularly within the active site, is similar to those of bacterial and eukaryal RNase Ps, suggesting a universal catalytic mechanism for all RNase Ps.

\footnotetext{
${ }^{1}$ State Key Laboratory of Molecular Biology, CAS Center for Excellence in Molecular Cell Science, Shanghai Institute of Biochemistry and Cell Biology, Chinese Academy of Sciences, Shanghai 200031, China. ${ }^{2}$ University of Chinese Academy of Sciences, Chinese Academy of Sciences, Shanghai 200031, China. ${ }^{3}$ School of Life Science and Technology, ShanghaiTech University, Shanghai 201210, China. ${ }^{4}$ Ninth People's Hospital, Shanghai Jiao Tong University School of Medicine, Shanghai 200125, China. ${ }^{5}$ Shanghai Institute of Precision Medicine, Shanghai 200125, China. ${ }^{6}$ Shanghai Key Laboratory of Translational Medicine on Ear and Nose diseases, Shanghai 200125, China. ${ }^{7}$ Key laboratory of Cell Differentiation and Apoptosis of Chinese Ministry of Education, Shanghai Jiao Tong University School of Medicine, Shanghai 200025, China. ${ }^{8}$ These authors contributed equally: Futang Wan, Qianmin Wang, Jing Tan. Correspondence and requests for materials should be addressed to P.L. (email: pengfeilan@shsmu.edu.cn) or to J.W. (email: wujian@shsmu.edu.cn)

or to M.L. (email: leim@shsmu.edu.cn)
} 
$\mathrm{R}$ ibonuclease $\mathrm{P}$ (RNase $\mathrm{P}$ ) and ribosome are the only two naturally occurring ribozymes that are present in all three kingdoms of life. RNase P is an ancient ribonucleoprotein (RNP) complex that catalyzes the maturation of the $5^{\prime}$ end of precursor tRNAs (pre-tRNAs) ${ }^{1-3}$. Bacterial RNase $\mathrm{P}$ is composed of a single small RNase $\mathrm{P}$ protein (RPP) in addition to the RNase P RNA (RPR) ${ }^{4}$. Based on the secondary structures, bacterial RPRs have been further classified into two subtypes, the most common type A for ancestral and type B for Bacillus ${ }^{5}$. Structural information of both bacterial A-type and B-type RPRs reveals that RPR consists of two independently folded domains, the catalytic domain (C-domain) and the specificity domain (S-domain), which play key roles in substrate cleavage and substrate binding, respectively $^{6-11}$. It has been found that bacterial RPR even the C domain alone possesses catalytic activity under high ionic strength condition or in the presence of spermidine in vitro ${ }^{12,13}$. But the sole protein subunit RPP is essential for enhancing the efficiency and fidelity of substrate recognition and cleavage under physiological conditions ${ }^{14-19}$.

In contrast to bacteria, archaeal and eukaryal nuclear RNase Ps have evolved considerably more complex protein subunits, four to five in archaeal and nine to ten in eukaryal enzymes ${ }^{2}$. Archaeal RNase $\mathrm{P}$ is an evolutionary intermediate with chimeric features of both bacterial and eukaryal nuclear enzymes, and thus serves as an excellent system to provide insights into the structural and functional alterations that accompanied the gradual transformation of an ancient catalytic RNA to a protein-rich $\mathrm{RNP}^{3,20}$. Archaeal RNase Ps can be further classified into three major types (A, M, and $\mathrm{P}$ ) on the basis of the secondary structural characteristics of their RPRs ${ }^{21-23}$. A-type RPR largely resembles bacterial RPRs and displays trace amount of catalytic activity in vitro, whereas M-type RPR diverges more from bacterial RPRs with less complex structure and has not been shown the ability as an RNA-only ribozyme ${ }^{24,25}$. Type $\mathrm{P}$ is a more extreme case in which the RPR is only about two thirds of its type $A$ and $M$ relatives, but surprisingly the RPR itself still retains the tRNA processing activity in vitro ${ }^{21}$.

Previous biochemical studies of in vitro reconstituted archaeal RNase Ps provide us the first glimpse of the roles of RPP subunits $24,26,27$. Single turn-over kinetic studies revealed that protein subcomplex (Pop5-Rpp30) ${ }_{2}$ is responsible for enhancing the cleavage rate of the tRNA precursors, while Rpp21-Rpp29 contributes to the increased substrate affinity ${ }^{28}$. Ribosomal protein L7Ae is the fifth subunit of archaeal RNase P and was shown to increase the thermostability of the Pyrococcus horikoshii RNase $\mathrm{P}$ holoenzyme $\mathrm{e}^{29,30}$. Although crystal structures of individual protein subunits and protein subcomplexes have been determined $^{29,31-38}$, their structural and enzymatic roles in archaeal RNase P holoenzyme still remain unclear.

Recently, mechanistic understanding of eukaryal RNase P has been taken a big step forward through the cryo-electron microscopy (EM) structures of the yeast and human RNase Ps, which reveal the spatial organization of eukaryal nuclear RNase Ps as well as the detailed mechanisms of substrate recognition and processing 39,40 . To gain insights into the structure and function of archaeal RNase P holoenzyme and its evolutionary relationships with bacterial and eukaryal enzymes, we reconstitute the RNase P holoenzyme from Methanocaldococcus jannaschii (Mtype) and determine its cryo-EM structure alone and in complex with a tRNA substrate. The structures fill a void for the structural insights into the RNase $\mathrm{P}$ evolution and provide mechanistic understanding of the catalysis of archaeal RNase P.

\section{Results}

In vitro reconstitution of the MjaRNase $\mathbf{P}$ holoenzyme. The MjaRNase P holoenzyme contains a 252-nucleotide RPR and five protein subunits Pop5, Rpp30, Rpp29, Rpp21, and L7Ae ${ }^{28,30,41}$. To reconstitute the MjaRNase P complex, we first over-expressed L7Ae as an individual subunit, Rpp29-Rpp21 and (Pop5-Rpp30) $)_{2}$ as heterodimeric and heterotetrameric subcomplexes in Escherichia coli, respectively (Supplementary Fig. 1a). The MjaRPR was transcribed and purified from an in vitro transcription system. However, RPR alone behaved poorly and tended to form soluble aggregates as revealed by size exclusion chromatography (SEC) analysis (Supplementary Fig. 1b). Notably, when purified L7Ae was added into the transcription reaction, RPR behaved properly as a monodispersed molecule, suggesting that L7Ae presumably functions as a chaperone for the correct folding and stability of RPR (Supplementary Fig. 1b). The in vitro transcribed RPR in the presence of L7Ae was sequentially mixed with purified Rpp29Rpp21 and (Pop5-Pop30) 2 subcomplexes and analyzed by SEC (Fig. 1a). Strikingly, addition of (Pop5-Rpp30) ${ }_{2}$ shifted the elution peak of the holoenzyme to a position with an apparent molecular weight of $\sim 440 \mathrm{kDa}$ that is about twice of the calculated molecular weight of MjaRNase P ( 210 kDa) (Fig. 1a), suggesting that the reconstituted MjaRNase P likely adopts a dimeric configuration that is mediated by the (Pop5-Rpp30) heterotetramer. Next, we employed negative staining EM to further examine the oligomeric state of the MjaRNase P complex, and found that the homogenous and monodispersed particles exhibited an elongated overall conformation (Fig. 1b). Consistent with the SEC analysis, two-dimensional class average of the particles confirmed that the reconstituted MjaRNase $\mathrm{P}$ holoenzyme is indeed a dimeric complex with a clear two-fold symmetry (Fig. 1b).

Careful analysis of the M. jannaschii genome identified 35 tRNA genes (Supplementary Table 1). Incubation of $M$. jannaschii pre-tRNA Arg with the reconstituted MjaRNase P holoenzyme leds to the cleavage of the $5^{\prime}$ leader from pre-tRNA Arg molecule in a magnesium ion $\left(\mathrm{Mg}^{2+}\right)$ dependent manner (Fig. 1c and Supplementary Fig. 2a, b). This result demonstrated that the in vitro reconstituted MjaRNase $\mathrm{P}$ complex is a fully functional enzyme. Notably, MjaRNase P can also efficiently process both human and $E$. coli pre-tRNAs (Supplementary Fig. 2a, b), consistent with the notion that RNase $\mathrm{P}$ recognizes the conserved structural feature, but not specific sequences of tRNA molecules ${ }^{39,40}$.

Overall architecture of $\mathbf{M j a R N a s e} \mathbf{P}$. To reveal the structure of MjaRNase P, the reconstituted MjaRNase P holoenzyme was subjected to cryo-EM analysis using a Falcon III direct camera, resulting in a well-defined electron density map of MjaRNase $\mathrm{P}$ at a resolution of $4.6 \AA$ (Fig. 2a, Supplementary Fig. 3 and Supplementary Table 2). To further understand how the tRNA substrate is recognized and processed by MjaRNase $\mathrm{P}$, we mixed MjaRNase $\mathrm{P}$ with $E$. coli pre-tRNA ${ }^{\mathrm{Tyr}}$ at a ratio of $1: 10$ and subjected the mixture to cryo-EM single particle analysis. Notably we obtained the three-dimensional reconstruction of MjaRNase P in complex with the mature form of the tRNA ${ }^{\text {Tyr }}$ substrate at a resolution of $4.3 \AA$ (Fig. 2a, Supplementary Fig. 4 and Supplementary Table 2). We speculated that the $5^{\prime}$ leader of pre-tRNA ${ }^{\text {Tyr }}$ was cleaved during EM sample preparation.

The EM reconstruction revealed that the MjaRNase $\mathrm{P}$ holoenzyme indeed adopts a dimeric conformation (Fig. 2a). The resolutions of the cryo-EM reconstruction were substantially improved by applying the two-fold symmetry, suggestive of a very rigid dimeric interface in the MjaRNase P complex (Supplementary Figs 3, 4). Secondary structural elements were clearly resolved in the EM density maps, allowing all folded domains 

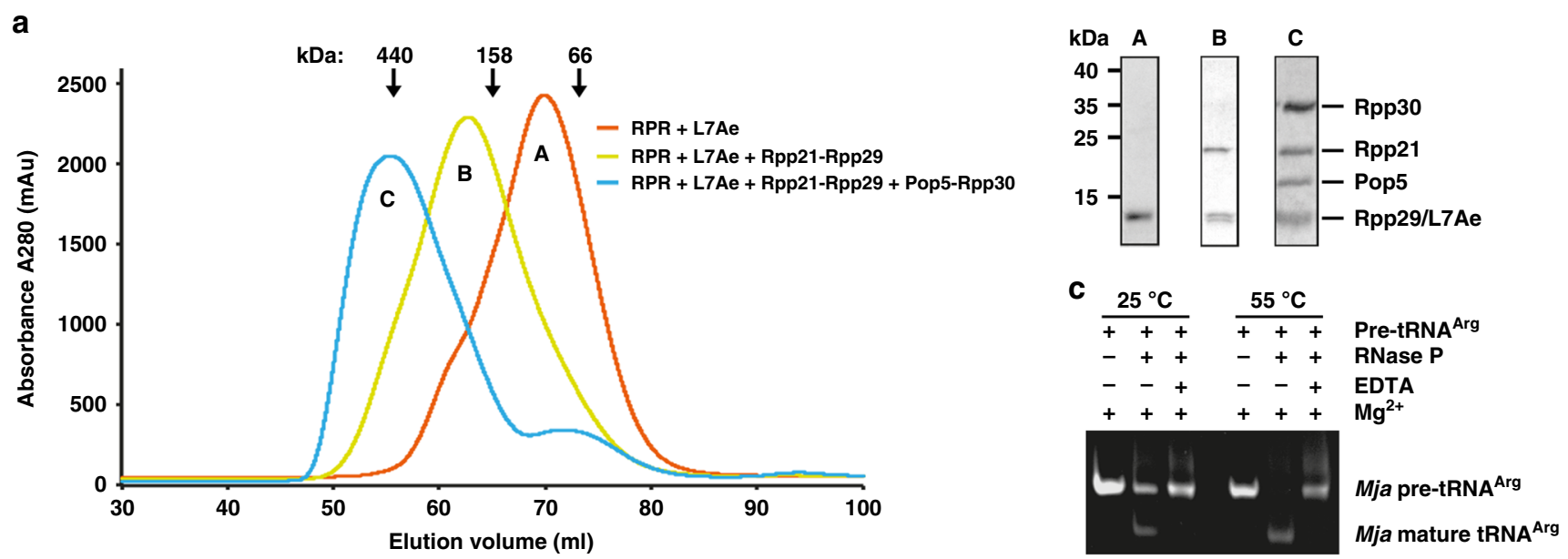

b
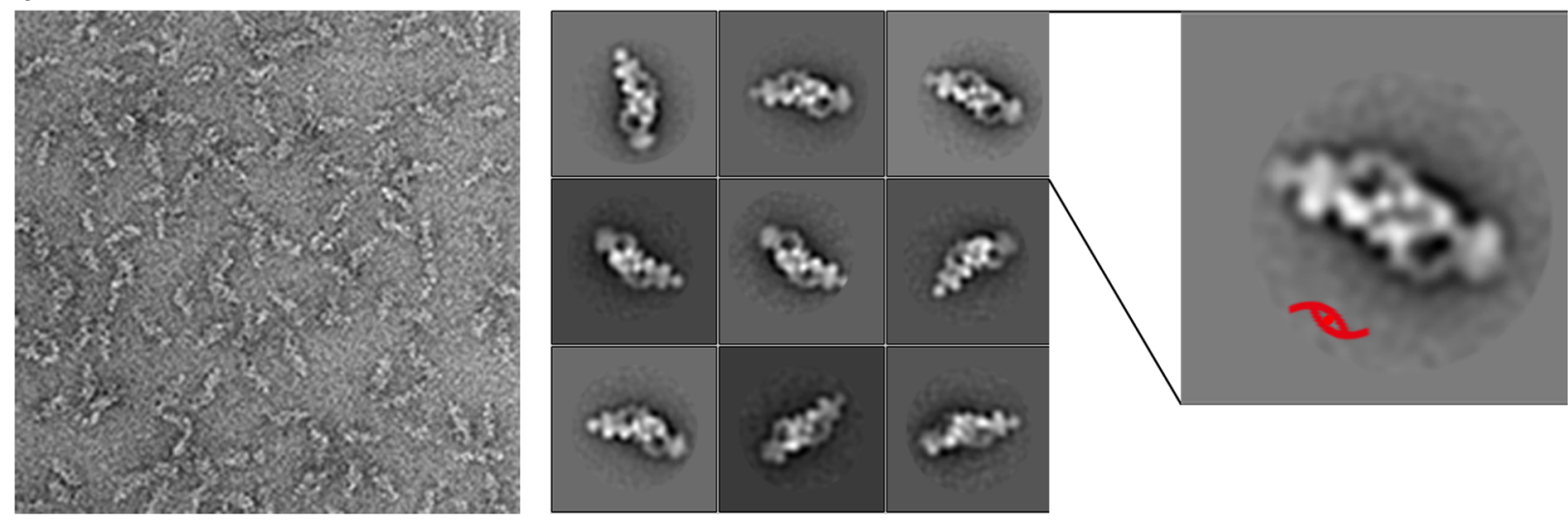

$50 \mathrm{~nm}$

Fig. 1 In vitro reconstitution of the MjaRNase P holoenzyme. a Size exclusion chromatographic profiles (left) and Coomassie-stained SDS-PAGE of the corresponding peaks in the profiles (right). b Negative staining EM analysis of MjaRNase P. Left: A representative negative-stained EM micrograph of MjaRNase P. Middle: Selected 2D class averages of MjaRNase P. Right: Close-up view of the 2D class averages with the C2 symmetry denoted a red symbol. c In vitro pre-tRNA ${ }^{A r g}$ processing assay of the MjaRNase P holoenzyme

predicted within MjaRNase P to be assigned (Supplementary Fig. 5). By using homologous structure modeling based on the crystal structures of bacterial RPR from Thermotoga maritima, archaeal components from $P$. horikoshii, and the cryo-EM structures of both yeast and human RNase P complexes, we generated an atomic model of the dimeric MjaRNase $\mathrm{P}$ holoenzyme, containing ten protein subunits and two catalytic RPRs (Fig. 2b).

The protein components of the MjaRNase P complex are arranged into an elongated configuration, which can be divided into three submodules, one (Pop5-Rpp30) ${ }_{2}$ heterotetramer at the center and two Rpp29-Rpp21-L7Ae heterotrimer at the ends (Fig. 2b). These protein submodules are intimately stringed together and serve as a long, extended holder to accommodate the two RPRs through a highly basic surface $\left(\sim 6500 \AA^{2}\right)$ (Fig. 2 c). The (Pop5-Rpp30 $)_{2}$ heterotetramer mediates symmetric interactions with two RPR molecules, which are organized into an extended, twisted Z-shaped configuration (Fig. 2b). Both termini of this extended RNA architecture associate with the Rpp29-Rpp21L7Ae heterotrimer (Fig. 2b). The structure of the MjaRNase P-tRNA complex shows that MjaRNase P adopts the same dimeric conformation as the apo structure with two tRNA substrates bound into each of the two active sites (Fig. 2b). Superposition analysis revealed that binding of the tRNA substrate only induces a $\sim 8^{\circ}$ change in the angle between the two MjaRNase $\mathrm{P}$ monomers, suggestive of a rigid dimeric MjaRNase P architecture (Fig. 2d).

M. jannaschii RPR. Similar to bacterial and eukaryal RPRs, MjaRPR can also be divided into two independently folded domains, the $\mathrm{C}$ and the $\mathrm{S}$ domains, with limited connections in between (Figs 3a, b) ${ }^{22,41}$. The $\mathrm{C}$ domain is composed of stems $\mathrm{P} 1$, $\mathrm{P} 2, \mathrm{P} 3, \mathrm{P} 4, \mathrm{P} 5$ and $\mathrm{P} 15$, and the $\mathrm{S}$ domain contains stems P7, P10, P12, P12.1, and P12.2 (Fig. 3a, b). The $\mathrm{C}$ and $\mathrm{S}$ domains are connected by the P5-P7 stems, and by the interaction between stems P1 and P9 (Fig. 3b). Universally conserved regions CR-I, CR-IV, and CR-V encompassed by stems P1, P2, P3, and P15 form the catalytic pseudo-knot motif (P4), occupying the center of the $\mathrm{C}$ domain (Fig. 3c). The other two conserved regions CR-II and CR-III between stems P10 and P12 fold into two interleaved T-loops for tRNA substrate binding (Fig. 3c) ${ }^{10,39,40,42}$. Consistent with previous predictions, both pseudo-knot and T-loop motifs are identical to those observed in bacterial and eukaryal RPRs (Supplementary Fig. 6), confirming that they are conserved structural features in RNase Ps among all three domains of life ${ }^{10,39,40}$. MjaRPR also contains an extension beyond stem P12, which folds back to form a characteristic kink-turn (K-turn) structure (Fig. 3b, c) $)^{30}$. Phylogenetic and secondary structural analyses revealed that an extension with a K-turn beyond stem 
a

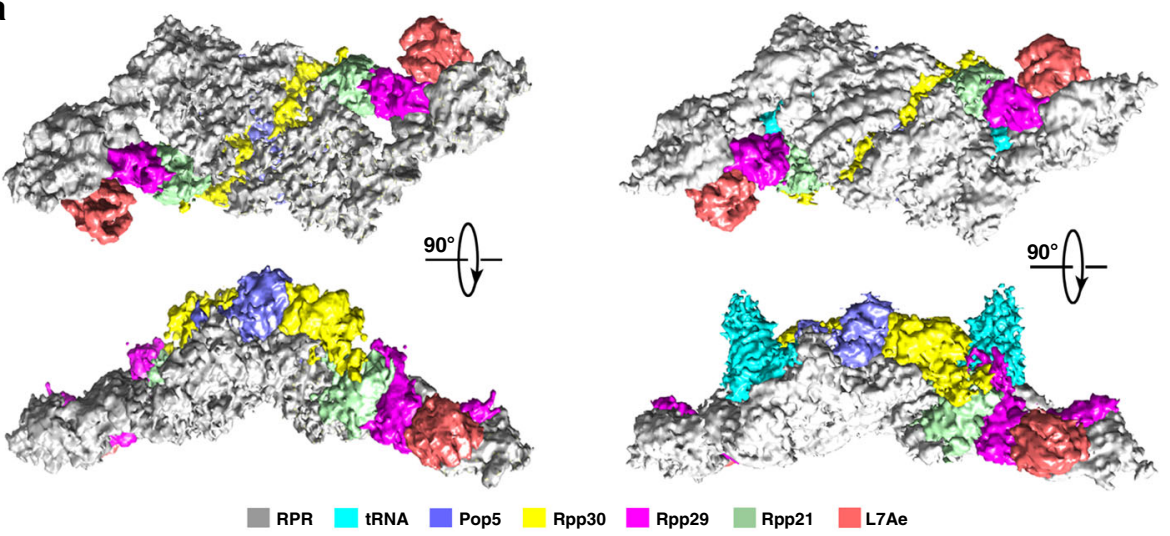

b
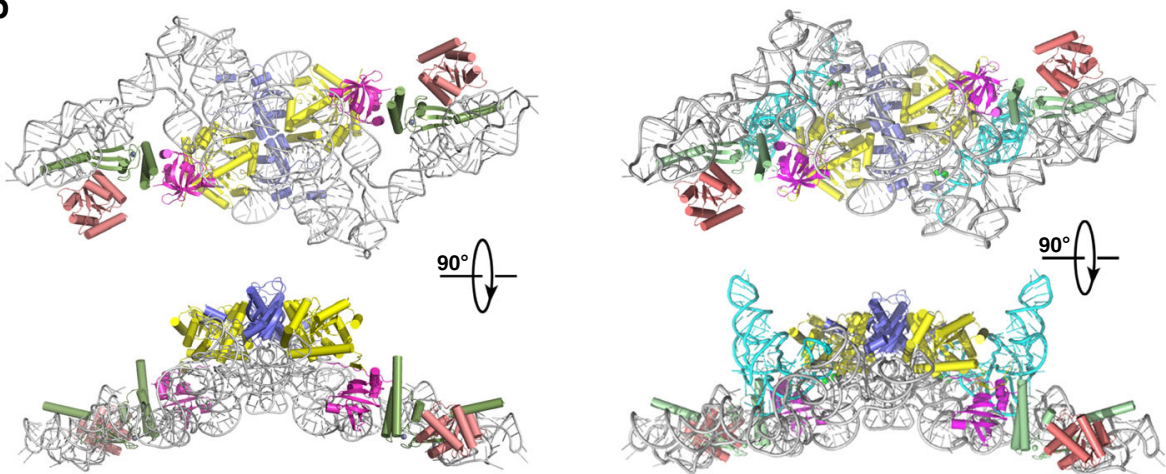

C

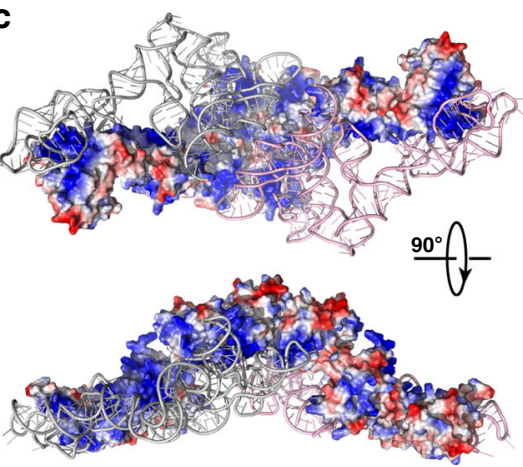

d

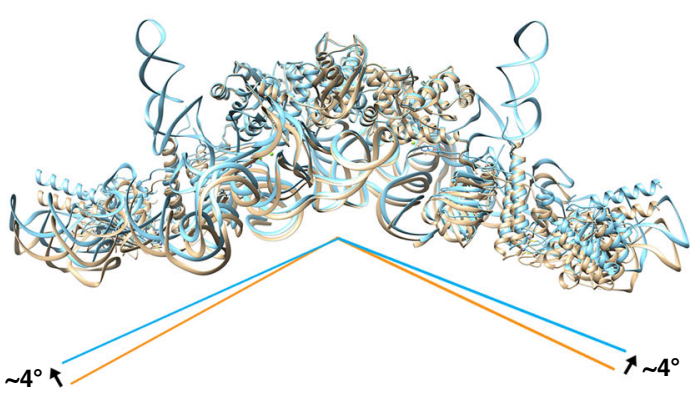

Fig. 2 Overall structures of the MjaRNase P complex with or without tRNA. a The cryo-EM density maps of MjaRNase P (left) and the MjaRNase P-tRNATyr complex (right) are shown in two orthogonal views. Protein and RNA components are color-coded and the scheme is shown below the figure. $\mathbf{b}$ Two orthogonal views of the atomic model of MjaRNase P (left) and the MjaRNase P-tRNA complex (right) are shown in cartoon representation. Protein and RNA components are color-coded as in (a). c Two orthogonal views of the surface electrostatic potential of the protein assembly in MjaRNase $\mathrm{P}$ reveals a continuous highly basic surface that binds two RPRs (negative: red; positive: blue). The two RPRs are colored in pink and gray, respectively. $\mathbf{d}$ Superposition of the structures of MjaRNase P with or without tRNA shows that tRNA binding only induces a $\sim 8^{\circ}$ change in the angle between the two MjaRNase $\mathrm{P}$ monomers. MjaRNase P and the MjaRNase P-tRNA complex are colored in wheat and palecyan, respectively

P12 is a conserved feature in most archaeal RNase P RNAs (Supplementary Fig. 7) 30,37,38,43,44.

MjaRNase P RPPs. Crystal structures of all protein components of $P$. horikoshii RNase $P$ have been determined $29,31-34,36$. Given the high sequence conservation between $P$. horikoshii and $M$. jannaschii RPPs (Supplementary Fig. 8), it is not surprising that the structures of individual protein components, the (Pop5Rpp30) $)_{2}$ heterotetramer and the Rpp29-Rpp21 heterodimer revealed in the MjaRNase $\mathrm{P}$ holoenzyme structure highly resemble previously determined $P$. horikoshii crystal structures (Supplementary Fig. 9). However, what is surprising is that in the holoenzyme one (Pop5-Rpp30) 2 heterotetramer and two
Rpp29-Rpp21-L7Ae heterotrimer are interlinked together to form a long extended decamer with a highly positively charged surface that holds two copies of MjaRPR (Figs 2c, 4a, b). A salient feature of this decamer architecture is the symmetric connection between the heterotetramer and two heterotrimers (Fig. 4a). The Cterminal long tail of Rpp30 sticks out of the main body of Rpp30 and folds into a $\beta$ strand to become the edge of the $\beta$ barrel of Rpp29, defining the key linkage in the protein decamer (Fig. 4c and Supplementary Fig. 10a). Moreover, the C-terminal short hydrophobic tail of Rpp29 extends out and fits into a hydrophobic groove of Rpp30, further strengthening the connection between the (Pop5-Rpp30) 2 heterotetramer and the Rpp29Rpp21-L7Ae heterotrimer (Fig. 4d and Supplementary Fig. 10b). Notably, except for a few A-type archaeal RNase Ps, the 
a

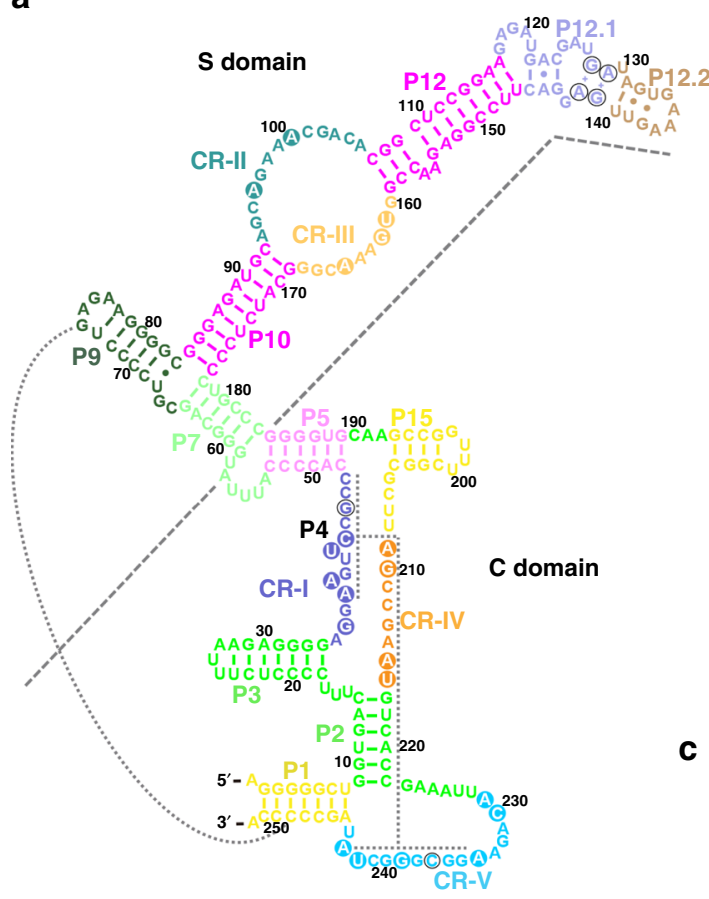

C b
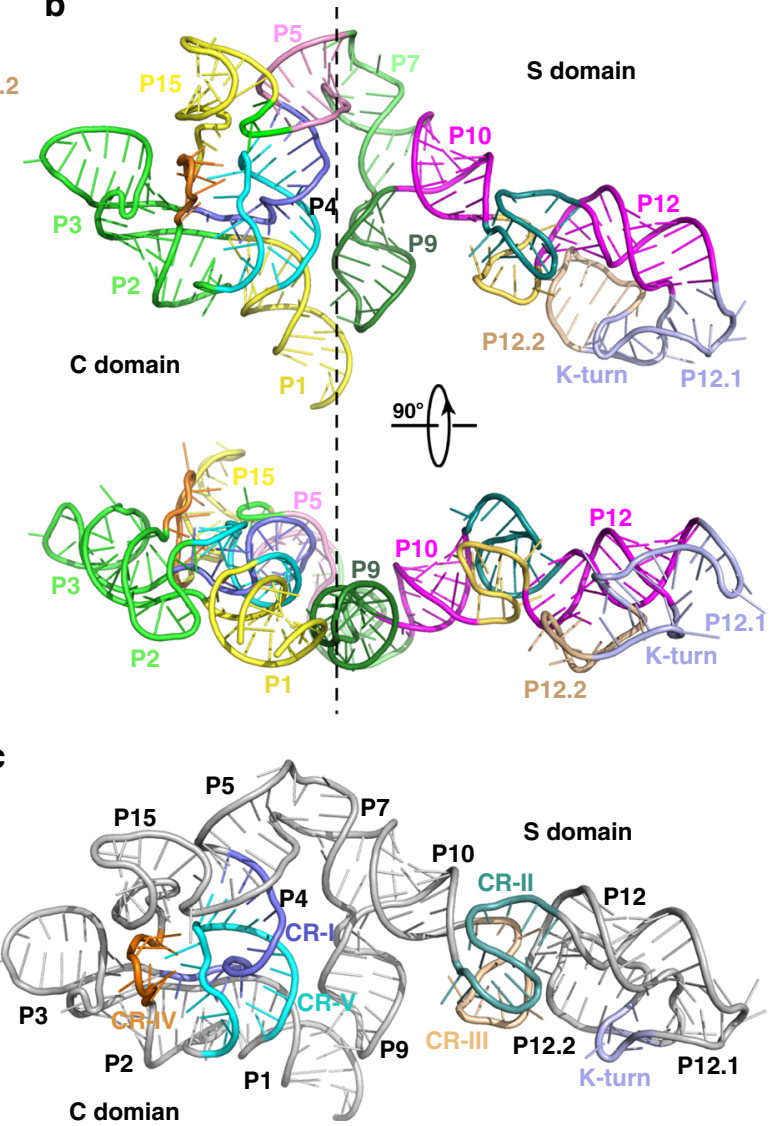

Fig. 3 Structure of the M. jannaschii RPR. a Secondary structure of RPR. Structural elements are colored to match their labels. The conserved regions of RPR (CR-I to CR-V) are colored in slate, deepteal, yelloworange, orange and cyan, respectively. The conserved P4 stem is denoted with dotted line. Dotted line between stem P1 and P9 indicates long-range RNA-RNA interaction. b Two orthogonal views of the overall structure of RPR. RPR is colored as in (a). c Overall structure of the RPR. The pseudoknot and the T-loop regions locate in the $\mathrm{C}$ and $\mathrm{S}$ domains of RPR, respectively. CR-I to CR-V are colored as in (a)

C-terminal tail of Rpp30 is highly conserved in most archaeal Rpp30 proteins (Supplementary Fig. 11), suggesting that the intermolecular connection between Rpp30 and Rpp29, and therefore, the overall architecture of the protein assembly is very likely conserved in most archaeal RNase P holoenzymes.

Another previously unobserved protein interaction is between Rpp21 and L7Ae. The L-shaped Rpp21 resides in the middle between Rpp29 and L7Ae, and the three proteins sequentially packs against one another to form the heterotrimer (Fig. 4c and Supplementary Fig. 12a). On one side, Rpp21 mediates extensive interactions with Rpp29 in a similar manner as their P. horikoshii homologs (Fig. 4c and Supplementary Fig. 9b) ${ }^{36}$. On the other side, the flat surface of Rpp21 holds L7Ae through both hydrophobic and electrostatic interactions so that L7Ae is in a suitable position to recognize the K-turn of MjaRPR (Fig. 4c and Supplementary Fig. 12b, c). The two interfaces bury exposed surface areas of $960 \AA^{2}$ and $690 \AA^{2}$ respectively, suggesting that the Rpp29-Rpp21 interaction is stronger than that between Rpp21 and L7Ae. Given both Rpp21 and L7Ae interact with $M j a \mathrm{RPR}$, it is likely that the protein-RNA interactions assist the formation of the Rpp29-Rpp21-L7Ae heterotrimer in the holoenzyme (Supplementary Fig. 12a). This is consistent with the fact that previous biochemical analysis failed to detect the interaction between Rpp21 and L7Ae $\mathrm{A}^{45,46}$.

In accordance with previous predictions, the MjaRNase $\mathrm{P}$ holoenzyme structure confirmed that $M$. jannaschii protein components are indeed structural homologs of their eukaryal counterparts (Supplementary Fig. 9a-c). It is noteworthy that archaeal Pop5 had evolved into two different eukaryal proteins
(Pop5 and Rpp14 in human RNase P) (Supplementary Fig. 9a) ${ }^{47}$. Consequently, the M. jannaschii (Pop5-Rpp30) ${ }_{2}$ heterotetramer became a Pop5-Rpp14-(Rpp30) ${ }_{2}$ heterotetramer with a 1:1:2 stoichiometry (Supplementary Fig. 9a) ${ }^{39}$. In addition, not only individual protein components are conserved, the intersubcomplex (Pop5-Rpp30) - $^{-}$Rpp29-Rpp21-L7Ae) connection mediated by Rpp30 and Rpp29 is also conserved in human RNase P holoenzyme structure (Fig. 4e) ${ }^{39}$. In contrast, none of the $\mathrm{MjaRNase}$ P protein components is structurally similar to the sole protein subunit RPP of bacterial RNase P (Supplementary Fig. 9a-d), suggesting that MjaRPPs are evolutionarily related to eukaryal RPPs, but not to bacterial RPP ${ }^{47}$.

RPP-RPR interactions. The (Pop5-Rpp30) $)_{2}$ heterotetramer locates in the center of the dimeric MjaRNase P holoenzyme (Fig. 5a). Pop5 adopts a typical RNA-recognition-motif fold, sitting on the junction between stems P2 and P3 of RPR (Fig. 5a). A highly basic cleft formed by the $\mathrm{N}$-terminal tail and C-terminal helices $\alpha 3$ and $\alpha 4$ of Pop5 tightly holds the zigzagged CR-IV of RPR (Fig. 5a). While Pop5 only contacts one RPR in the complex, Rpp30 is involved in electrostatic interactions with both RPR molecules (Fig. 5b). Together, the symmetric contacts between Rpp30 and RPR stems P2-P3 and between Pop5 and CR-IV of RPR mold MjaRNase P into a dimeric holoenzyme complex (Fig. 5a, b).

At the distal ends of the MjaRNase $\mathrm{P}$ complex, the heterotrimer Rpp29-Rpp21-L7Ae associates with the terminal regions of stems $\mathrm{P} 1$ and $\mathrm{P} 9$ of $\mathrm{RPR}$, and the highly basic Rpp21 
a

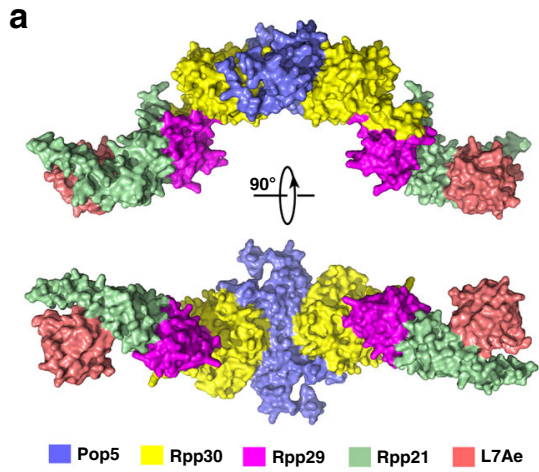

b

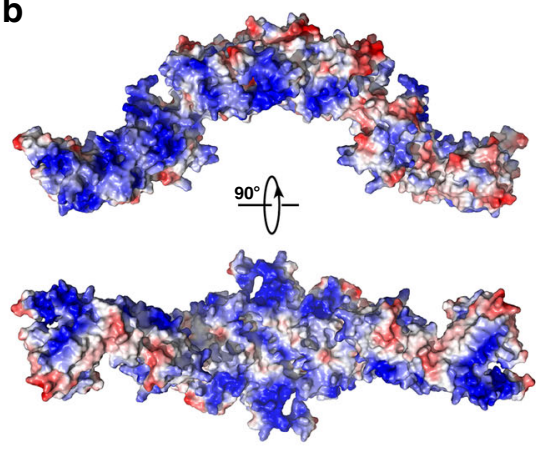

d

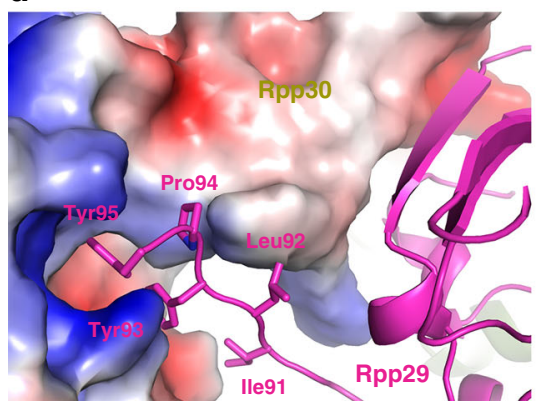

c

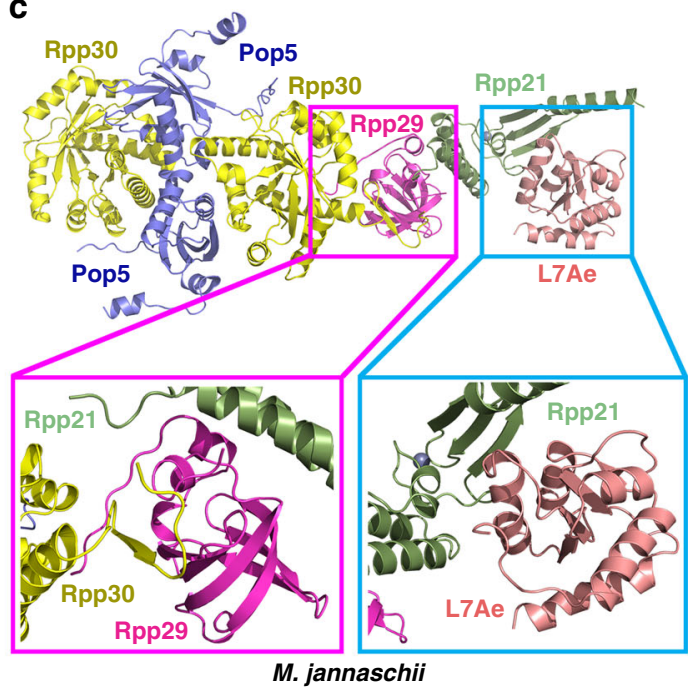

$\mathbf{e}$

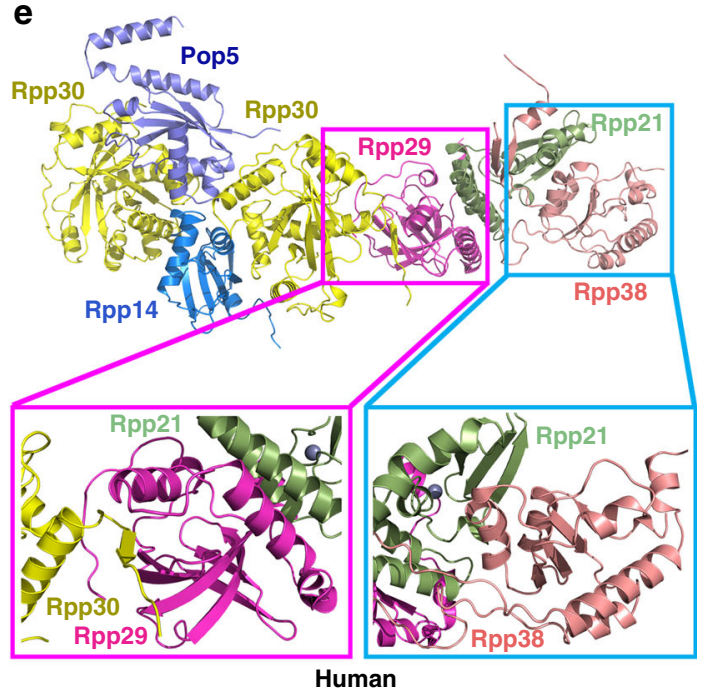

Fig. 4 Protein components of MjaRNase P. a Two orthogonal views of the MjaRNase P protein assembly is shown in surface representation. Protein components are color-coded and the scheme is shown below the figure. $\mathbf{b}$ Two orthogonal views of the electrostatic surface potential of the protein assembly (negative: red; positive: blue). c Cartoon representation of the Mja (Pop5-Rpp30) 2 -Rpp29-Rpp21-L7Ae heptamer. Protein components are colored as in (a). Bottom left: close-up view of the interface between Rpp30 and Rpp29. Bottom right: close-up view of the interface between Rpp21 and L7Ae. $\mathbf{d}$ The C-terminal tail of Rpp29 binds into a hydrophobic groove of Rpp30. Rpp30 is shown in electrostatic surface representation and Rpp29 is shown in cartoon. e Cartoon representation of the human Pop5-Rpp14-(Rpp30) ${ }_{2}$-Rpp29-Rpp21-Rpp38 heptamer. Bottom left: close-up view of the interface between Rpp30 and Rpp29. Bottom right: close-up view of the interface between Rpp21 and Rpp38

makes extensive interactions with a large area of the RPR S domain, including the T-loops, stem P12 and the extension beyond P12 (Fig. 5c and Supplementary Fig. 12b). L7Ae specifically recognizes the K-turn and helps stem P12.1 fold back onto P12 (Fig. 5c and Supplementary Fig. 12c). Together, two interlinked protein subcomplexes (Pop5-Rpp30) ${ }_{2}$ and Rpp29Rpp21-L7Ae form a single extended protein assembly that presumably helps stabilize the relative positions of the $\mathrm{C}$ and $\mathrm{S}$ domains of the RPR (Figs 2b, 4c).

tRNA recognition. Although pre-tRNA ${ }^{\mathrm{Tyr}}$ was used in the cryoEM analysis of the MjaRNase P-tRNA ${ }^{\text {Tyr }}$ complex, the $\sim 4.0-\AA$ resolution at the catalytic center suffices to show that the $5^{\prime}$ leader of tRNA is absent in the structure, indicating that the tRNA molecule in the complex is the mature tRNA product after cleavage (Supplementary Fig. 13a). The MjaRNase P-tRNA complex structure reveals that MjaRNase P employs a doubleanchor mechanism to accommodate the coaxially stacked acceptor arm and $\mathrm{T} \psi \mathrm{C}$ arm of tRNA into the substrate-binding pocket (Fig. 6a). In the S domain of RPR, CR-II and CR-III fold into two interleaved T-loops to form one of the anchor (referred to as the T-loop anchor) to stack with the $\mathrm{T} \psi \mathrm{C}$ and $\mathrm{D}$ loops of tRNA, securing the corner of the L-shaped tRNA in the substrate pocket of MjaRNase P (Fig. 6b and Supplementary Fig. 13b). On the other end of the acceptor arm of tRNA, the central A191 (referred to as the A anchor) in the three-nucleotide linker $\mathrm{L}_{5-15}$ between stems P5 and P15 of RPR packs on the first base-pair G1-C81 of the acceptor stem of tRNA, anchoring the cleavage site of tRNA right at the catalytic center of MjaRNase P (Fig. $6 \mathrm{c}$ and Supplementary Fig. 13c). The two RNA anchors respectively locate in the $\mathrm{C}$ and $\mathrm{S}$ domains of RPR, functioning as a measuring device to recognize the coaxially stacked acceptor and $\mathrm{T} \psi \mathrm{C}$ arms of tRNA substrates, which measure a fixed distance of 12 base 
a

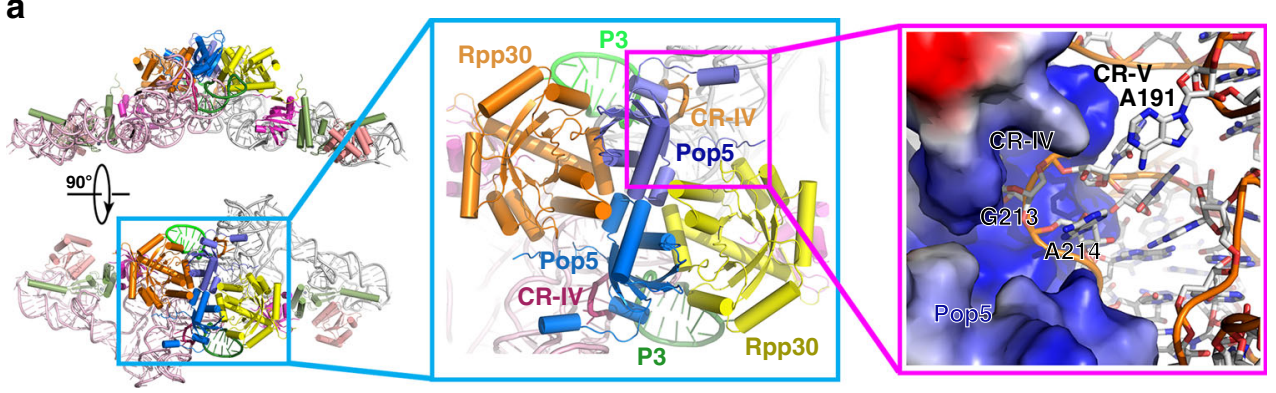

b

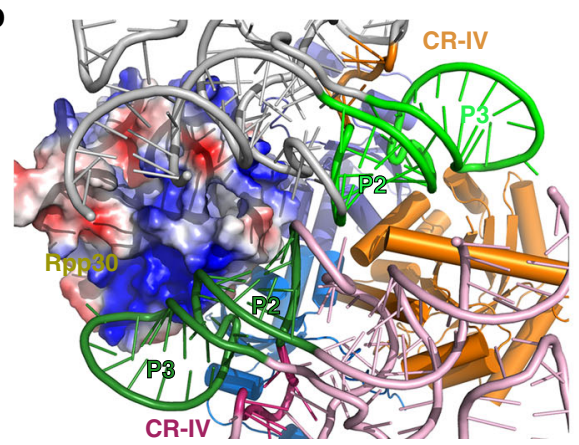

C

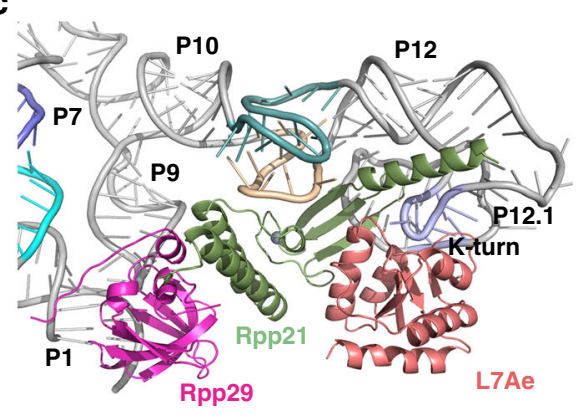

Fig. 5 Interaction between RPR and RPPs in MjaRNase P. a Left: The atomic model of the MjaRNase P complex is shown in two orthogonal views. RPPs are color-coded as in Fig. 2a. The two RPR molecules are colored in pink and gray, respectively. Middle: Close-up view of the interface between two RPRs (colored in pink and gray) and the (Pop5-Rpp30) 2 heterotetramer. The two P3 stems are colored in deepteal and green, respectively. Right: Close-up view of the interface between Pop5 and CR-IV of RPR. Pop5 is shown in electrostatic surface representation and RPR in cartoon representation.

b Rpp30 simultaneously interacts with the two RPR molecules in the dimeric holoenzyme. One Rpp30 is shown in electrostatic surface representation and the interacting P2-P3 stem from one RPR is colored in deepteal and the other interacting RPR in green. c Close-up view of the interface between the Rpp29Rpp21-L7Ae heterotrimer and RPR. RPR and RPPs are shown in cartoon and colored as in Fig. 3c and Fig. 2a, respectively

pairs in all tRNA molecules (Fig. 6a) ${ }^{1,10,48-52}$. This double-RNAanchor for tRNA recognition is conserved in most archaeal and bacterial RPRs $4,23,53,54$.

In addition to the two anchors, the $3^{\prime}$-RCCA sequence of bacterial tRNAs is also recognized by a conserved RNA element, loop $\mathrm{L}_{15}$, in most bacterial RPRs through base-pairing interactions (Supplementary Fig. 14) ${ }^{10}$. Database search and sequence analysis revealed that some $M$. jannaschii pre-tRNAs do not contain a RCCA sequence at their $3^{\prime}$ termini (Supplementary Fig. 15). This observation is in accordance with the fact that MjaRPR lacks a $3^{\prime}$-RCCA recognition element in its small terminal loop at stem P15 (Fig. 3a), suggesting that MjaRNase P might have lost this tRNA recognition element during evolution. Indeed, although we used a tRNA with a $3^{\prime}$-RCCA sequence in the MjaRNase P-tRNA complex structure, only the first two nucleotides A82 and C83 of the RCCA motif can be modeled into the major groove of the short P15 stem of RPR in the EM density, whereas both C84 and A85 are not visible, presumably disordered in the MjaRNase P-tRNA complex structure (Supplementary Fig. 16). Therefore, it is unlikely the $3^{\prime}$-RCCA is recognized by MjaRPR through base-pair interactions as in bacterial RNase Ps.

Comparative analysis revealed that MjaPop5 and its eukaryal homologs occupy the same location on their respective RPRs and hold the zigzagged CR-IV of RPR in their deep basic clefts in the same manner (Fig. 6d). In the yeast RNase P-tRNA complex structure, Pop5 stabilizes CR-IV to make direct stacking interactions with nucleobases at the $-1,-2$, and -3 positions of the $5^{\prime}$ leader of pre-tRNA (Fig. 6d and Supplementary Fig. 17) ${ }^{40}$. The close structural resemblance between MjaPop5 and yeast Pop5 suggests that it is very likely MjaPop5 employs the same mechanism to recognize the $5^{\prime}$ leader of pre-tRNAs (Fig. $6 \mathrm{~d}$ ). In addition to Pop5, other protein components of MjaRNase P also make direct contributions to tRNA binding. Rpp30, Rpp29 and
Rpp21 form a continuous highly basic surface that is complementary to the L-shaped tRNA, burying $\sim 890 \AA^{2}$ interface area between the tRNA and proteins (Fig. 6a). Consistent with this observation, previous biochemical studies showed that a tworesidue mutation of PhoRpp29 at this interface substantially reduced the tRNA processing activity of PhoRNase P (Supplementary Fig. 18) ${ }^{36}$.

tRNA processing. The cryo-EM density of the MjaRNase PtRNA complex allowed unambiguous placement of the $5^{\prime}$ end of the mature tRNA in the catalytic center, which resides at the junction between CR-I and CR-V of MjaRPR (Fig. 7a and Supplementary Fig. 13a). The spatial arrangement of MjaRPR nucleotides around the $5^{\prime}$ end of tRNA, including G40, U41, A233, and A234 as well as the universally conserved uridine U42 in stem P4, highly resembles those observed in T. maritima and the newly reported yeast and human RNase P-tRNA complex structures (Fig. 7a) ${ }^{10,39,40}$. In the yeast RNase $P$ structure in complex with a pre-tRNA substrate, equivalent nucleotides A91, U92, U93, G343, and A344 coordinates two catalytic $\mathrm{Mg}^{2+}$ ions (Fig. 7a $)^{40}$. Consistent with this structural resemblance, single nucleotide deletion of $\mathrm{U} 42(\Delta \mathrm{U} 42)$ or replacement with an adenosine (U42A) greatly diminished the enzymatic activity of $M j a$ RNase P, strongly supporting that MjaRPR nucleotides at the active site likely play the same role in coordinating two $\mathrm{Mg}^{2+}$ ions essential for catalysis (Fig. 7b). Based on the highly conserved architecture at the catalytic center, we propose that the chemical nature of pre-tRNA processing is evolutionarily conserved from bacteria to archaea to eukarya. In this mechanism, an evolutionarily conserved RNA architecture coordinates two $\mathrm{Mg}^{2+}$ ions at the catalytic center, one of which (M1) facilitates a hydroxyl ion to perform an $\mathrm{S}_{\mathrm{N}} 2$-type nucleophile attack at the cleavage site of the pre-tRNA substrate, whereas the other (M2) stabilizes the 

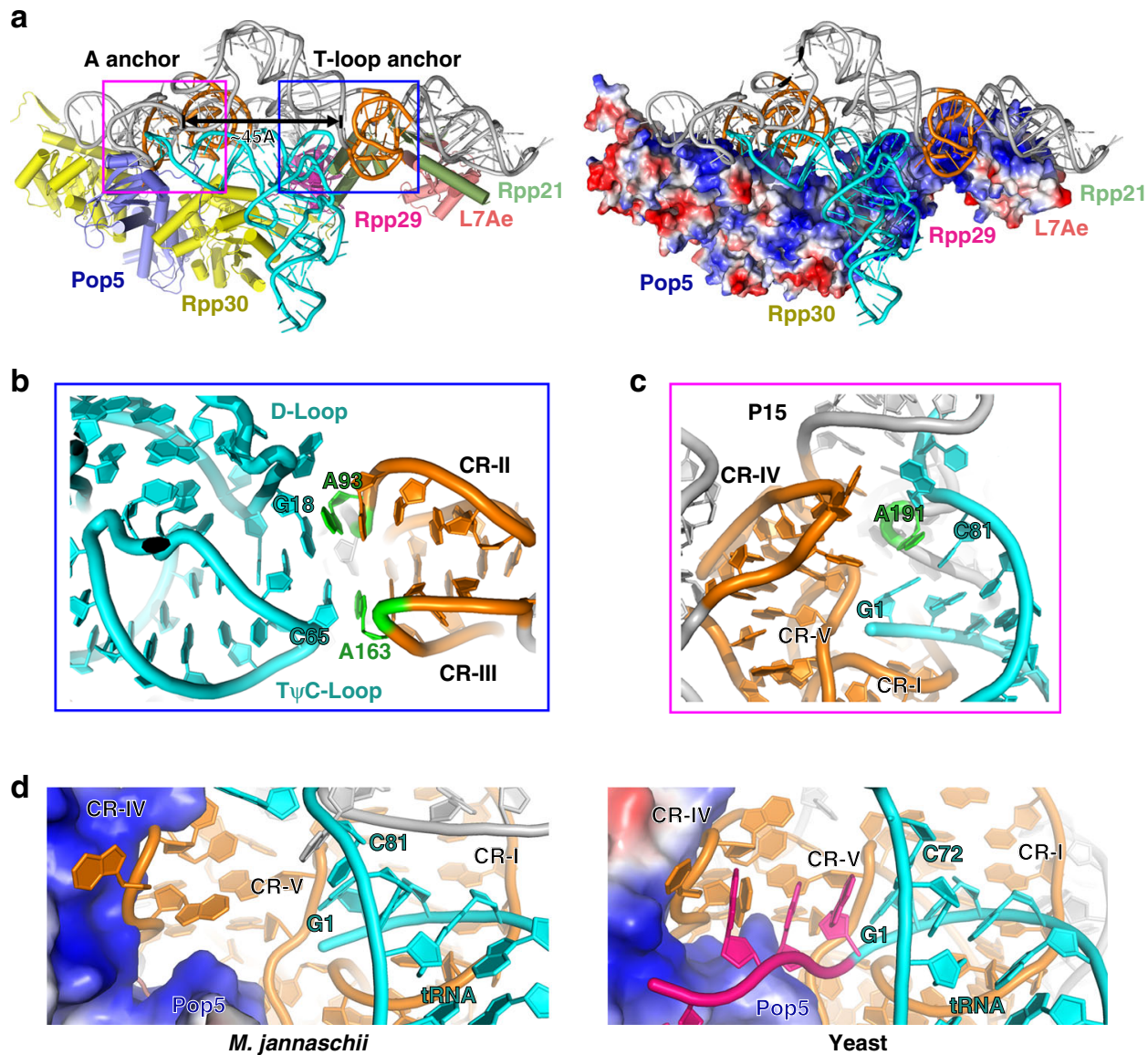

Fig. 6 tRNA recognition by MjaRNase P. a Overall structure of the monomeric MjaRNase P complex bound with tRNA. Protein components are shown in cartoon (left) and in electrostatic surface (right) representations, respectively. The magenta box denotes the $A$ anchor and the blue box denotes the T-loop anchor. The distance between the two anchors is highlighted with a black double arrow. The tRNA is colored in cyan. $\mathbf{b}$ Close-up view of the TyC and D loops of tRNA that stack with the T-loop anchor in CR-II and CR-III of RPR. c Close-up view of the central nucleotide A191 (in green) in loop L5-15 of RPR that stacks on the first base-pair G1-C81 of tRNA. $\mathbf{d}$ Left panel: Close-up view of the active site in the MjaRNase P-tRNA complex. Right panel: Close-up views of the active site in the yeast RNase P-pre-tRNA complex (PDB: 6AH3). Pop5 is shown in surface representation and colored in electrostatic potential. RPR and tRNA are shown in cartoon and colored in orange and cyan, respectively. The $5^{\prime}$ leader of yeast pre-tRNA ${ }^{\text {Phe }}$ is colored in magenta

transition state and mediate proton transfer to the $3^{\prime}$ scissile oxygen (Fig. 7c) ${ }^{55-63}$.

Dimeric organization of MjaRNase P. A surprising observation from the MjaRNase P holoenzyme structure is that it adopts a dimeric conformation with a two-fold symmetry (Fig. 2a). The (Pop5-Rpp30) ${ }_{2}$ heterotetramer sits at the center of the MjaRNase P complex and organizes the dimerization (Fig. 5a). Two Pop5 proteins symmetrically recognize two CR-IV regions so that the loops between stems P2 and P4 (nucleotides 223-228) from the two RPR molecules staggered pack together (Fig. 8a). In human and yeast RNase P monomeric structures, the symmetric Mja(Pop5-Rpp30) heterotetramer is replaced by non-symmetric Pop5-(Rpp30) $2^{-}$ Rpp14 and Pop5-(Rpp30) 2 -Pop8 heterotetramer respectively, in which only Pop5 is cable of binding CR-IV of the RNAs (Supplementary Fig. 9a) ${ }^{39,40}$. In addition, in both human and yeast RNase $\mathrm{P}$ complexes, there is a P19 stem inserted in the loop between stems $\mathrm{P} 2$ and $\mathrm{P} 4$, which would cause a severe collision if the RNase P complex contained two RNA molecules in a similar manner as in MjaRNase $\mathrm{P}^{39,40}$. These structural features are conserved in all eukaryal RNase Ps, suggesting that eukaryal RNase Ps should adopt a monomeric but not dimeric configuration.

It is noteworthy that the (Pop5-Rpp30) 2 heterotetramer binds to the C domain of both RPR molecules symmetrically in the MjaRNase P dimeric complex, so that Rpp30 is involved in tRNA binding in one monomeric complex while sitting on the short P3 stem of RPR from the other complex (Fig. 8b). Markedly, the equivalent Rpp30 that contact the tRNA substrate in both human and yeast monomeric RNase P complexes is also buttressed by additional protein subunit and/or RPR element (Supplementary Fig. 19). Structurally, these interactions appear to stabilize Rpp30 for complex assembly and tRNA substrate binding (Fig. 8b and Supplementary Fig. 19). Consistent with this idea, alanine substitution of PhoRpp30 $0^{\text {Lys196 (equivalent to MjaRpp30 }}{ }^{\text {Lys198) }}$ ) at the interface between Rpp30 and the second RPR in the dimer greatly reduced the pre-tRNA cleavage activity, suggesting that the dimeric conformation of archaeal RNase $\mathrm{P}$ is crucial for its enzymatic activity (Fig. $8 \mathrm{~b})^{34}$. To further examine the function of dimerization, we designed a monomeric mutant MjaRPR with an artificial P19 stem inserted between nucleotides G223 and A224, which should preclude the dimer formation (Supplementary Fig. 20). Indeed, in vitro reconstitution with this mutant RPR resulted in a monomeric MjaRNase P complex as revealed by both gel filtration and negative staining EM analyses (Fig. 8c, d). In vitro activity assay showed that this monomeric mutant MjaRNase P exhibited substantially reduced pre-tRNA processing activity (Fig. 8e), underscoring the importance of dimerization in the in vitro activity of MjaRNase P. Whether the MjaRNase P holoenzyme adopts a dimeric conformation in vivo is still unclear and warrants further investigations. 
a

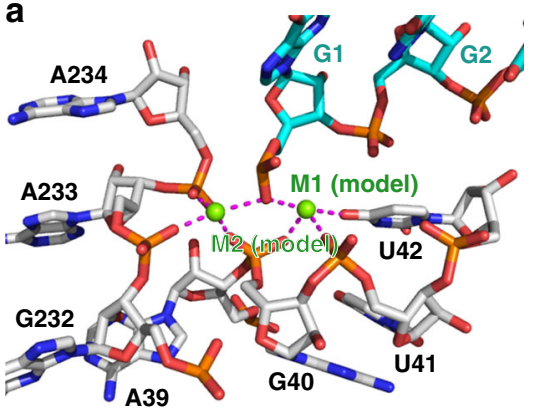

M. jannaschii
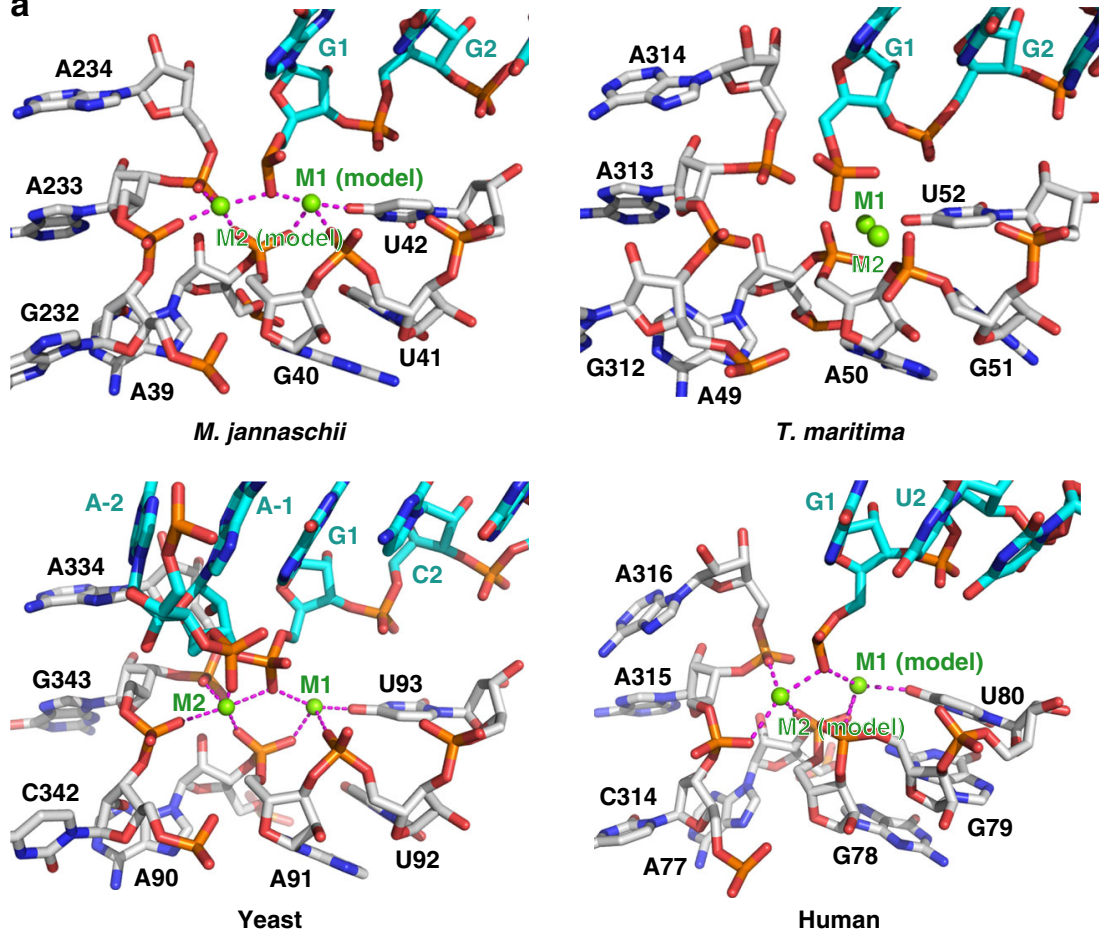

T. maritima

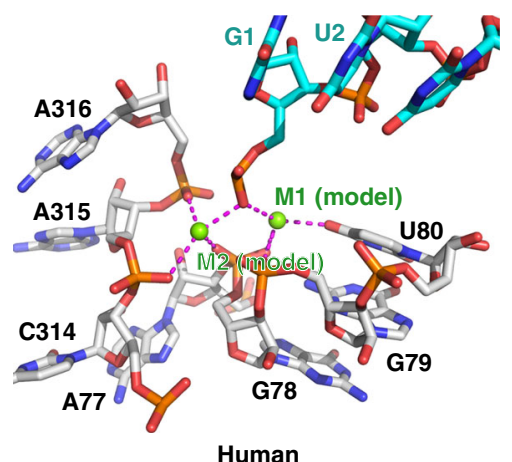

b
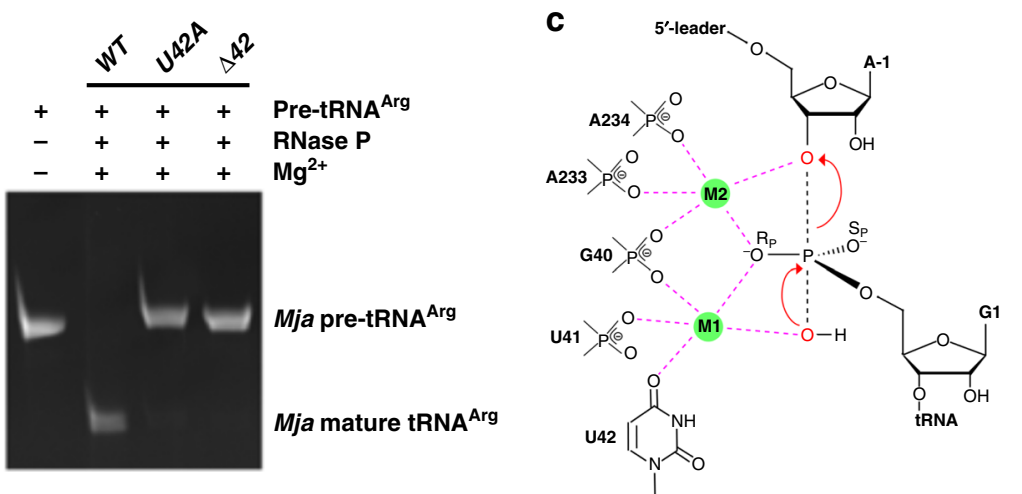

Fig. 7 The catalytic center of MjaRNase P. a Close-up views of the catalytic centers of MjaRNase P (top left), T. maritima RNase P (top right, PDB: $3 Q$ Q1Q), yeast RNase P (bottom left, PDB: 6AH3), and human RNase P (bottom right, PDB: 6AHU). Two catalytic $\mathrm{Mg}^{2+}$ ions (M1 and M2) in the active site of MjaRNase P and human RNase P shown in green spheres were modeled based on the yeast RNase P-tRNA complex structure. RPR and tRNA are showed in stick representation and colored in silver and cyan, respectively. The coordination of the two $\mathrm{Mg}^{2+}$ ions is denoted by magenta dashed lines. $\mathbf{b}$ In vitro pretRNA processing assay of the MjaRNase P holoenzyme reconstituted with WT, U42A and $\Delta U 42$ RPR, respectively. c Proposed reaction mechanism for $5^{\prime}$ leader cleavage of pre-tRNA by MjaRNase P. The reactive oxygens are colored in red, the pre-tRNA scissile phosphate is depicted in a transition state, and the interactions between catalytically important nucleotides and reactive oxygens mediated by $\mathrm{Mg}^{2+}$ ions (M1 and M2) are shown as magenta dashed lines

\section{Discussion}

Archaea have been widely used to study the evolution of many biological processes from prokaryotes to eukaryotes. The structure of the MjaRNase P holoenzyme reported here provides us a unique opportunity to understand the evolution of RNase P, which accompanied the gradual transformation from an ancient catalytic RNA to a protein-rich RNP.

Although $\mathrm{MjaRNase} \mathrm{P}$ is distinct from bacterial and eukaryal RNase Ps, it contains important structural features from both. MjaRNase P employs two RNA anchors, the T-loop anchor and the $\mathrm{A}$ anchor in the $\mathrm{S}$ and $\mathrm{C}$ domains respectively, for tRNA substrate recognition (Figs 6a and 9). This RNA-based apparatus is conserved in the majority bacterial and archaeal RNase

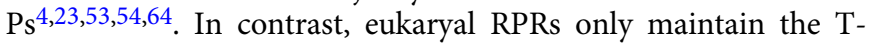
loop anchor, and the $\mathrm{A}$ anchor is replaced with a protein one mediated by a eukaryal specific protein Pop1 (Fig. 9) ${ }^{39,40}$. In all cases, the two anchors must be stabilized with a fixed distance that is optimal for accommodating the coaxially stacked acceptor and $\mathrm{T} \psi \mathrm{C}$ arms of pre-tRNA substrates (Fig. $6 \mathrm{a})^{10,39,40}$. In bacterial RPR, auxiliary RNA elements mediate long-range RNARNA interactions to stabilize the tertiary RNA structure so that the distance between the two anchors is optimal for tRNA binding (Fig. 9 and Supplementary Fig. 6a) ${ }^{10}$. However, MjaRPR lacks most of these auxiliary RNA elements (Figs 3a, 9). Consequently, in contrast to bacterial RPRs, the $\mathrm{C}$ and the $\mathrm{S}$ domains of $M j a \mathrm{RPR}$ are only loosely connected and require newly evolved protein assembly (Pop5-Rpp30) ${ }_{2}$ (Rpp29-Rpp21-L7Ae) to stabilize the RPR for tRNA binding and processing (Figs 6a, 9). This protein-aided stabilization mechanism is faithfully inherited by eukaryal RNase Ps (Fig. 9) ${ }^{39,40}$. Together, these observations 
a

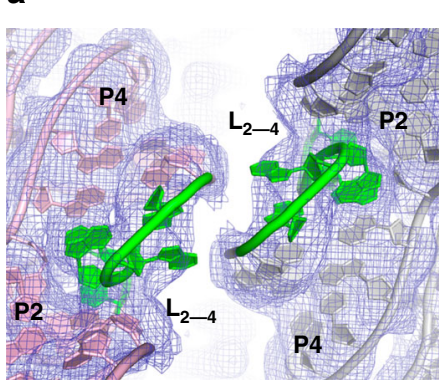

C

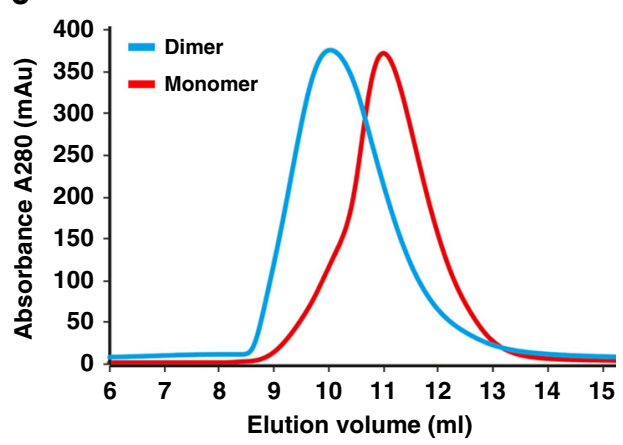

d

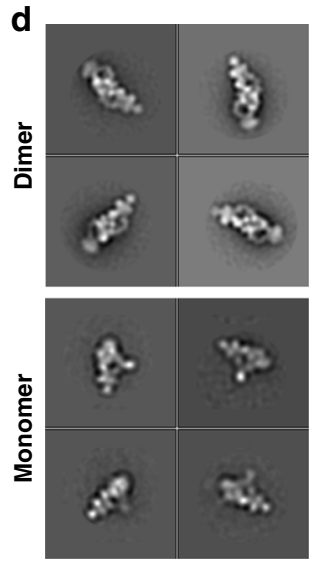

b

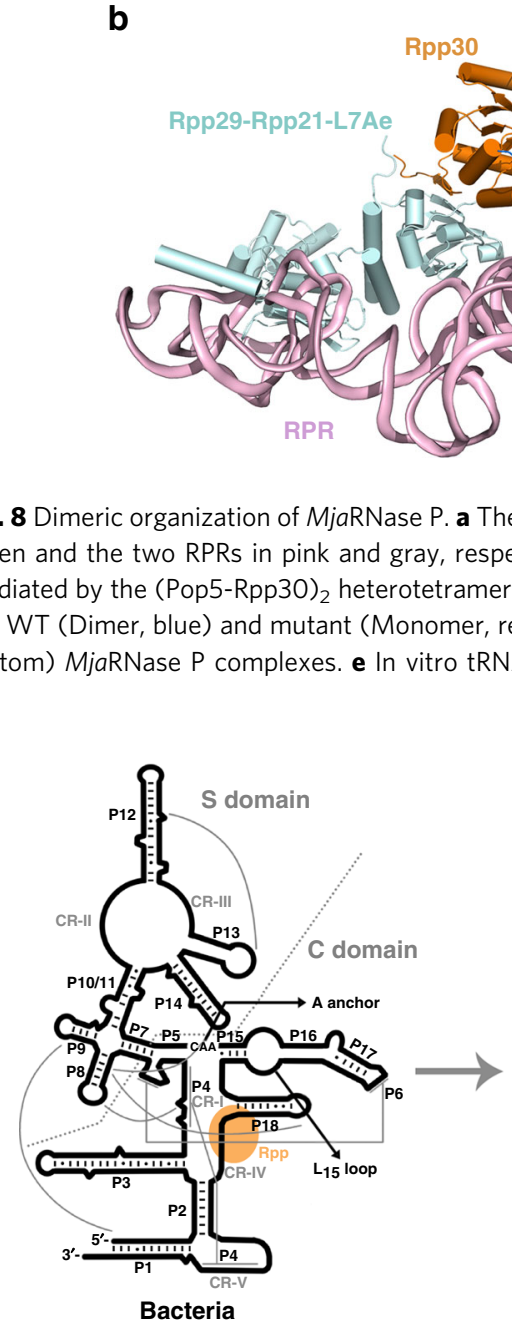

Pop5

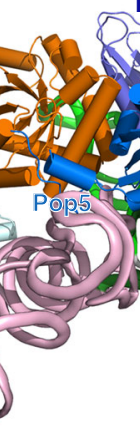

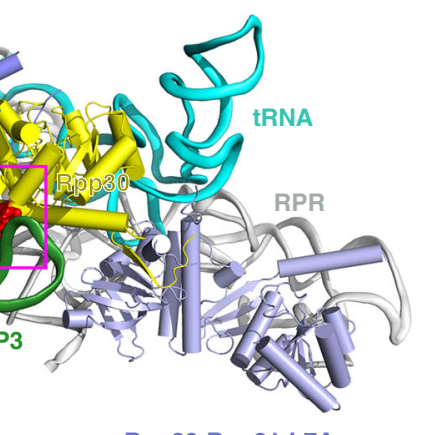

e

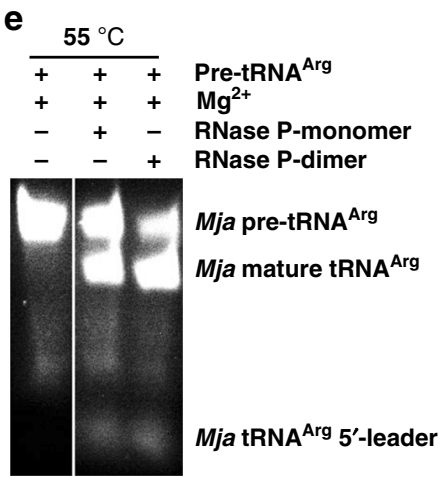

Fig. 8 Dimeric organization of MjaRNase P. a The loops between stems P2 and P4 from the two RPRs staggered pack together. The two loops are colored in green and the two RPRs in pink and gray, respectively. The EM density map is shown in light blue mesh. $\mathbf{b}$ Overall view of the dimeric conformation mediated by the (Pop5-Rpp30) 2 heterotetramer. Rpp30 simultaneously interacts with the two RPR molecules in the dimeric holoenzyme. c SEC profiles of the WT (Dimer, blue) and mutant (Monomer, red) MjaRNase P complexes. d Selected 2D class averages of the WT (Dimer, top) and mutant (Monomer, bottom) MjaRNase P complexes. e In vitro tRNA processing assay of WT (Dimer) and mutant (Monomer) MjaRNase P complexes
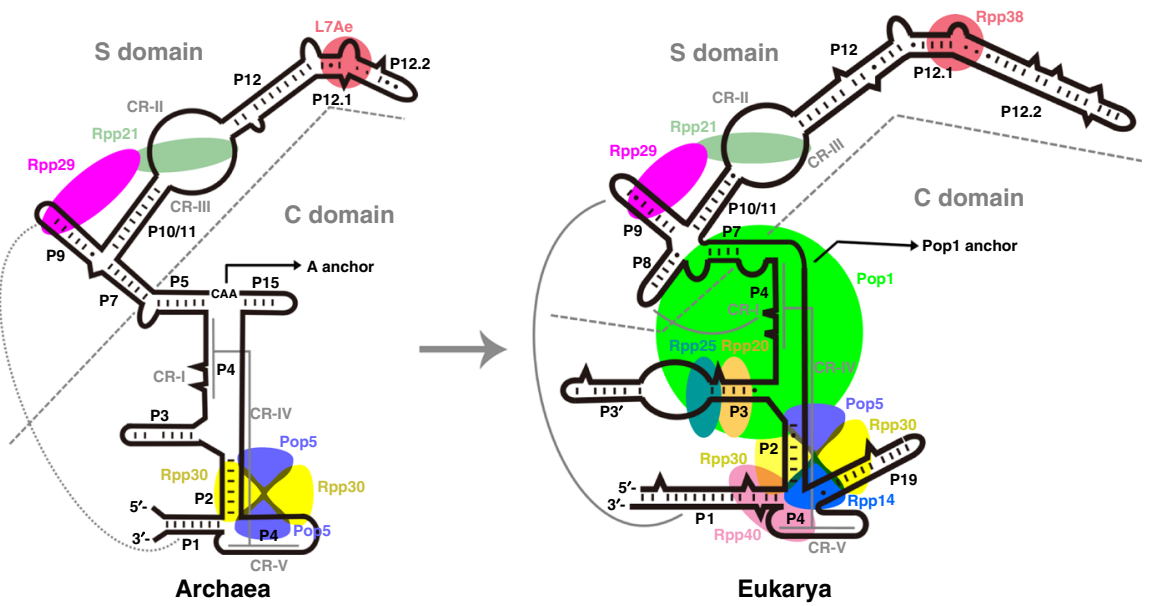

Fig. 9 A evolution model of RNase P from bacteria to archaea, and to eukarya. Secondary structure models of T. maritima, M. jannaschii and human RNase P RNAs are based on their atomic structures. Long-range RNA-RNA interactions are denoted by gray lines. Protein components are colored as in Fig. 2a and are placed in positions based on the atomic structures

demonstrate that MjaRNase $\mathrm{P}$ is a hybrid of bacterial and eukaryal RNase Ps, with bacterial-like two RNA-based anchors but eukaryal-like protein-aided stabilization mechanism, exemplifying that archaea are evolutionary intermediates between bacteria and eukarya.

Bacterial RPR element including loop $\mathrm{L}_{15}$ that forms specific base pairs with the $3^{\prime}$-RCCA sequence of tRNA substrates is absent in MjaRPR and other M-type RNase Ps (Fig. 3a and
Supplementary Figs $6 a, 14)^{10,22,23,41}$. Therefore, similar to yeast and human RNase Ps, the $3^{\prime}$-RCCA of tRNA is not a recognition element in archaeal M-type RNase Ps. It is noteworthy that the $3^{\prime}$-RCCA recognition element is conserved in some A-type archaeal RPRs, and that all P. horikoshii tRNA genes contain the $3^{\prime}$ RCCA sequence (Supplementary Fig. 15) 23,54 . It is plausible that PhoRNase P might still maintain the $3^{\prime}$-RCCA recognition mechanism and therefore is evolutionarily closer to bacterial 
RNase Ps than other archaeal RNase Ps. Structural information of the PhoRNase P holoenzyme is needed to test this hypothesis.

P-type archaeal RNase $\mathrm{P}$ is a radically minimal form of RNase $\mathrm{P}^{21}$. It only contains a $\mathrm{C}$ domain but lacks a recognizable $\mathrm{S}$ domain ${ }^{21}$, suggesting that this type of archaeal RNase P does not have a T-loop anchor as A-type and M-type RNase Ps. In addition, the $\mathrm{L}_{5-15}$ loop between stems P5 and P15 in some P-type RPRs only contains two but not three nucleotides ${ }^{21}$ so that P-type RNase Ps might also do not have a canonical A anchor. Notably, the complementary region of loop $\mathrm{L}_{15}$ that could pair with the $3^{\prime}$ RCCA sequence of tRNA still exists in P-type RPRs ${ }^{21}$. But, some P-type tRNA genes do not contain the $3^{\prime}$-RCCA sequence (Supplementary Fig. 15). Therefore, it is not clear whether P-type RNase $\mathrm{P}$ recognizes the tRNA substrate using the $3^{\prime}$-RCCA mechanism. Notwithstanding these dramatic changes, RPRs from P-type RNase Ps are still catalytically active in vitro ${ }^{21}$. The mystery behind P-type archaeal RNase P warrants further mechanistic investigation and structural characterization.

\section{Methods}

Protein expression and purification. Full-length genes of $M j a \mathrm{Pop} 5$ and $M j a$ Rpp30 were respectively cloned into the two multiple cloning sites of the pETDuet vector with a $6 \times$ His tag at the N-terminus of MjaRpp30 and were expressed in E. coli BL21 (DE3) cells. After induction for $18 \mathrm{~h}$ with $0.1 \mathrm{mM}$ IPTG at $18^{\circ} \mathrm{C}$, the cells were harvested and the pellets were resuspended in buffer $\mathrm{A}$ (50 mM Tris- $\mathrm{HCl}, \mathrm{pH} 8.0,1 \mathrm{M} \mathrm{NaCl}$ and $0.8 \mathrm{M}$ Urea). The cells were then lysed by sonication and the cell debris was removed by centrifugation. The supernatant was mixed with Ni-NTA agarose beads (Qiagen) and rocked for $4 \mathrm{~h}$ at $4{ }^{\circ} \mathrm{C}$ before elution with buffer B (50 mM Tris-HCl, pH 8.0, $1 \mathrm{M} \mathrm{NaCl}$ and $500 \mathrm{mM}$ imidazole). The protein elution was heated at $65^{\circ} \mathrm{C}$ for $30 \mathrm{~min}$ and the contaminants were removed by ultracentrifugation. The proteins were further purified by ionexchange and SEC. The purified proteins in buffer C (50 mM Hepes, $\mathrm{pH} 7.5$, $50 \mathrm{mM} \mathrm{MgCl} 2,200 \mathrm{mM} \mathrm{NaCl}, 500 \mathrm{mM} \mathrm{KCl}, 50 \mathrm{mM}$ potassium acetate) were concentrated to $\sim 20 \mathrm{mg} \mathrm{mL}^{-1}$ and stored at $-80^{\circ} \mathrm{C}$. The same procedure was used to express and purify MjaL7Ae and the MjaRpp29-MjaRpp21 heterodimer.

RNA oligonucleotides. The RPR was generated by in vitro run-off transcription with T7 RNA polymerase at $37^{\circ} \mathrm{C}$. During the transcription, the purified MjaL7Ae was added as a chaperone to help stabilize the newly transcribed $M j a$ RPR. The RPR was ultracentrifuged to remove the precipitants and then further purified by SEC with a Superdex200 column (GE Life Science) equilibrated with buffer $\mathrm{C}$ and was concentrate to $\sim 10 \mathrm{mg} \mathrm{mL}^{-1}$. The MjaRPR mutants were transcribed and purified similarly. The E. coli pre-tRNA ${ }^{\mathrm{Tyr}}$, M. jannaschii pre-tRNA ${ }^{\mathrm{Arg}}$, and human pretRNA $^{\text {Val }}$ were prepared as described above.

\begin{abstract}
MjaRNase P holoenzyme complex assembly. Biochemical reconstitution of the MjaRNase P holoenzyme was performed by incubating MjaRPR with the protein components with a molar ratio of $1: 3$ in buffer $\mathrm{C}$ at $37^{\circ} \mathrm{C}$ for $30 \mathrm{~min}$, and then at $55^{\circ} \mathrm{C}$ for another $30 \mathrm{~min}$. The $\mathrm{Mj} a \mathrm{RN}$ ase P holoenzyme was then purified by SEC with a Superdex 200 column (GE Life Science) equilibrated with buffer C. The purified MjaRNase $\mathrm{P}$ holoenzyme was concentrate to $\sim 5 \mathrm{mg} \mathrm{mL}^{-1}$. MjaRNase P was mixed with $E$. coli pre-tRNA ${ }^{\text {Tyr }}$ in a molar ratio of 1:10 to generate the MjaRNase P-pre-tRNA complex sample for EM experiments.
\end{abstract}

pre-tRNA processing assays. To characterize the activity of the reconstituted WT and mutant $M j a$ RNase P holoenzyme, pre-tRNA substrate was mixed with MjaRNas P holoenzyme with a molar ratio of $100: 1$ in $10 \mu \mathrm{L}$ buffer $\mathrm{C}$ at $25^{\circ} \mathrm{C}$ or $55^{\circ} \mathrm{C}$ for $30 \mathrm{~min}$. The reactions were quenched by adding the loading dye. The samples were then loaded into a $15 \%$ urea denaturing polyacrylamide gel with TBE buffer and stained with ethidium bromide.

Electron microscopy. The reconstituted MjaRNase P complex was first studied by the negative-staining EM. Copper grid coated with a thin carbon film was glowdischarged for $20 \mathrm{~s}$ and $5 \mu \mathrm{L}$ sample was applied onto it. The sample was incubated for $1 \mathrm{~min}$ and stained with $1 \%(\mathrm{w} / \mathrm{v})$ uranyl formate for another minute. About 300 micrographs were taken in Tecnai G2 Spirit microscope operated at $120 \mathrm{kV}$ and recorded on a $4 \mathrm{k} \times 4 \mathrm{k}$ CCD camera with a defocus of $\sim-2 \mu \mathrm{m}$ and a magnification of $67,000 \times\left(1.74 \AA \mathrm{pixel}^{-1}\right)$.

Cryo-EM samples were prepared with Vitrobot Mark IV (FEI) at $4{ }^{\circ} \mathrm{C}$ and $100 \%$ humidity. Ted Pella lacey copper grids coated with a thin layer of continuous carbon film were glow-discharged for $15 \mathrm{~s}$ and $4 \mu \mathrm{L}$ of the sample was applied (blotting parameters: waiting time $20 \mathrm{~s}$, blot force -1 , blot time $4 \mathrm{~s}$ ) and plungefrozen in liquid ethane. Since the sample was in a high-salt buffer, which hinders the cryo-EM data acquisition, the high-salt buffer was exchanged to a buffer contains $200 \mathrm{mM}$ salt ( $40 \mathrm{mM}$ Hepes, $\mathrm{pH} 7.5,200 \mathrm{mM} \mathrm{NaCl}$ ) on the grids quickly before plunge. For the MjaRNase P-tRNA complex, cryo-EM data was acquired on a Titan Krios (FEI) operated at $300 \mathrm{keV}$ equipped with a K2 Summit direct detector. The detector was operated in the super-resolution mode at $120,000 \times$ magnification with a calibrated physical pixel size of $1.32 \AA$. Data acquisition was carried out with SerialEM ${ }^{65}$ to record 4144 movies with a defocus range from -1.5 to $-2.5 \mu \mathrm{m}$ and with a total exposure time of $8 \mathrm{~s} .32$ frames were collected per movie at a dose rate of $6.2 \mathrm{e}^{-} \AA^{-2} \mathrm{~s}^{-1}$, which provided a total accumulated dose of $50 \mathrm{e}^{-} \AA^{-2}$. For the MjaRNase P holoenzyme, data were collected using the Folcan III camera in counting mode at a magnification of $81,000 \times\left(1.09 \AA\right.$ pixel $\left.^{-1}\right) .2611$ movies with defocus values ranging from -1.5 to $-2.5 \mu \mathrm{m}$ were acquired using EPU software with a total exposure time of $69 \mathrm{~s} .32$ frames were collected per movie with a total accumulated dose of $40 \mathrm{e}^{-} \AA^{-2}$.

Image processing. For negative staining analysis, $\sim 10,000$ particles were picked using e2box.py from EMAN $2^{66}$. Then all the subsequent steps of extraction, classification and initial model building were performed in RELION 2.167. The initial model was used for 3D refinement, which result in a map with a $23-\AA$ resolution. The map of the negative-stained MjaRNase P was low-pass filtered to $60 \AA$ and used for cryo-EM data processing.

For cryo-EM analysis of the MjaRNase P-tRNA complex dataset, we applied MotionCor $2^{68}$ to perform the frame alignment and dose-weighting, and Gctf ${ }^{69}$ to estimate the contrast transfer function (CTF) parameters. An initial set of approximately 2,000 particles were manually picked, then $2 \mathrm{D}$ class averages were calculated and used as reference for automatic picking with Gautomatch (http:// www.mrc-lmb.cam.ac.uk/kzhang/Gautomatch/). About 930,000 particles were auto-picked and selected for further 2D classification, which yielded a dataset containing about 829,000 particles. Then all particles were subjected to 3D refinement using a $60 \AA$-low pass filtered negative-stained map as a reference. The refined model was used as reference for 3D classification. The major class with reasonable features containing about 15,0000 particles was applied for further 3D refinement. Since MjaRNase P appears as a dimeric complex, the C2 symmetry was applied during $3 \mathrm{D}$ refinement, producing a final map at a $4.3-\AA \AA$ resolution based on the gold-standard FSC cut-off criterion at 0.143 . The density map was sharpened by applying a negative temperature factor automatically estimated by postprocessing program of RELION 2.167. Local resolution estimates were determined using RELION 2.167. For cryo-EM analysis of the apo MjaRNase P dataset, image processing procedures were the same as described above.

Model building. De novo atomic model building and rigid-docking of homologous structures are combined to build the model of the entire MjaRNase P complex with or without tRNA. Model building of the $\mathrm{C}$ domain of MjaRPR was mostly based on the bacterial RPR structure ${ }^{9,10}$, whereas the S domain mostly based on the human RPR structure ${ }^{39}$. We first modeled the characteristic three coaxially stacked RNA stems with standard double-stranded RNAs based on the bacterial RNase P structure (PDB: 3Q1Q). Then the conserved structure of CR-I-II-III-IV-V regions were identified and the models were fit into the EM density according to the bacterial and human RNase P, respectively (PDB: 3Q1Q and 6AHU). Next we built the K-turn by fitting the Pho P12.1 and P12.2 (PDB: 5DCV; 5XTM; 5Y7M) into the EM density. After assignment of all these elements, junction regions and singlestranded loops were then built manually with stereochemistry considerations. RNA nucleotide register was judged by conserved and unique regions of RPR, including the CR-I-IV-V pseudo-knot, CR-II-III T-loop anchor, the A-anchor and the Kturn. The final RPR structure satisfies the requirement of all these special regions as well as all the helical regions. The crystal structure of $E$. coli tRNA ${ }^{\text {Tyr }}$ was determined previously (PDB: 4V8D) ${ }^{70}$. We fit this crystal structure into the EM density with minor adjustment.

As for RPPs, we built their models based on the crystal structures of $M$. jannaschii and $P$. horikoshii RNase P protein subunits ${ }^{34,36,71}$. The crystal structure of $M j a \mathrm{~L} 7 \mathrm{Ae}$ has already been determined (PDB: $1 \mathrm{SDS})^{71}$. We directly fit $\mathrm{MjaL} 7 \mathrm{Ae}$ structure into the EM density with minor adjustment. For other protein components, we first fit each Pho proteins (PDB: 3WZ0; 2ZAE) into the EM density by rigid-body docking followed by manual adjustment and de novo model building for regions that are different from Pho protein structures. There are four major differences between $M j a$ and Pho protein structures. First, $M j a$ Rpp30 has an extra $\beta$ strand docking on MjaRpp29. Second, the C-terminal tail of $M j a R p p 29$ sticks out to fit into a hydrophobic groove of $M j a R p p 30$ whereas the Cterminal tail is missing in the PhoRpp29 structure. There are clear electron densities corresponding to these extra regions in the EM density map (Supplementary Fig. 10a, b). It is these extra structural elements of MjaRpp30 and MjaRpp29 that mediates the connection between the Mja (Pop5-Rpp30) heterotetramer and the Mja Rpp29-Rpp21-L7Ae heterotrimer (Fig. 4c). The third difference is that MjaRpp21 contains extra C-terminal residues that fold into a helix packing on both MjaL7Ae and the junction between stems P12 and P12.1/ P12.2 of MjaRPR (Fig. 5c and Supplementary Figs 5, 9b, 12a). Another difference is from MjaPop5, which has a short extra helix packing on the small terminal loop of stem P15 of MjaRPR (Fig. 4c and Supplementary Figs 5, 9b). The EM density clearly shows the extra density of this helix (Supplementary Figs 5, 12a). 
Reporting summary. Further information on research design is available in the Nature Research Reporting Summary linked to this article.

\section{Data availability}

The data that support the findings of this study are available from the corresponding author upon reasonable request. The cryo-EM 3D maps of the MjaRNase P holoenzyme and the MjaRNase P-tRNA complex were deposited in EMDB database with accession codes EMD-9899 and EMD-9900, respectively. The atomic models were deposited in $\mathrm{PDB}$ with accession codes $6 \mathrm{~K} 0 \mathrm{~A}$ and $6 \mathrm{~K} 0 \mathrm{~B}$, respectively.

Received: 11 August 2018 Accepted: 9 May 2019

Published online: 13 June 2019

\section{References}

1. Altman S., Kirsebom L. A. Ribonuclease P. The RNA world Second edition (Cold Spring Harbor Laboratory Press, Cold Spring Harbor, NY, 1999).

2. Esakova, O. \& Krasilnikov, A. S. Of proteins and RNA: the RNase P/MRP family. RNA 16, 1725-1747 (2010)

3. Walker, S. C. \& Engelke, D. R. Ribonuclease P: the evolution of an ancient RNA enzyme. Crit. Rev. Biochem. Mol. Biol. 41, 77-102 (2006).

4. Kazantsev, A. V. \& Pace, N. R. Bacterial RNase P: a new view of an ancient enzyme. Nat. Rev. Microbiol. 4, 729-740 (2006).

5. Haas, E. S., Banta, A. B., Harris, J. K., Pace, N. R. \& Brown, J. W. Structure and evolution of ribonuclease P RNA in Gram-positive bacteria. Nucleic Acids Res. 24, 4775-4782 (1996).

6. Krasilnikov, A. S., Yang, X., Pan, T. \& Mondragon, A. Crystal structure of the specificity domain of ribonuclease P. Nature 421, 760-764 (2003).

7. Krasilnikov, A. S., Xiao, Y., Pan, T. \& Mondragon, A. Basis for structural diversity in homologous RNAs. Science 306, 104-107 (2004).

8. Kazantsev, A. V. et al. Crystal structure of a bacterial ribonuclease P RNA. Proc. Natl Acad. Sci. USA 102, 13392-13397 (2005).

9. Torres-Larios, A., Swinger, K. K., Krasilnikov, A. S., Pan, T. \& Mondragon, A. Crystal structure of the RNA component of bacterial ribonuclease P. Nature 437, 584-587 (2005).

10. Reiter, N. J. et al. Structure of a bacterial ribonuclease P holoenzyme in complex with tRNA. Nature 468, 784-789 (2010).

11. Loria, A. \& Pan, T. Domain structure of the ribozyme from eubacterial ribonuclease P. RNA 2, 551-563 (1996).

12. Mao, G. et al. Critical domain interactions for type A RNase P RNA catalysis with and without the specificity domain. PLoS ONE 13, e0192873 (2018).

13. Guerrier-Takada, C., Haydock, K., Allen, L. \& Altman, S. Metal ion requirements and other aspects of the reaction catalyzed by M1 RNA, the RNA subunit of ribonuclease P from Escherichia coli. Biochemistry 25, 1509-1515 (1986).

14. Sun, L., Campbell, F. E., Zahler, N. H. \& Harris, M. E. Evidence that substratespecific effects of $\mathrm{C} 5$ protein lead to uniformity in binding and catalysis by RNase P. EMBO J. 25, 3998-4007 (2006).

15. Niranjanakumari, S., Stams, T., Crary, S. M., Christianson, D. W. \& Fierke, C. A. Protein component of the ribozyme ribonuclease $\mathrm{P}$ alters substrate recognition by directly contacting precursor tRNA. Proc. Natl Acad. Sci. USA 95, 15212-15217 (1998).

16. Sun, L. \& Harris, M. E. Evidence that binding of C5 protein to P RNA enhances ribozyme catalysis by influencing active site metal ion affinity. RNA 13, 1505-1515 (2007).

17. Sun, L., Campbell, F. E., Yandek, L. E. \& Harris, M. E. Binding of C5 protein to $\mathrm{P}$ RNA enhances the rate constant for catalysis for P RNA processing of pretRNAs lacking a consensus $(+1) / C(+72)$ pair. J. Mol. Biol. 395, 1019-1037 (2010).

18. Green, C. J., Rivera-Leon, R. \& Vold, B. S. The catalytic core of RNase P. Nucleic Acids Res. 24, 1497-1503 (1996).

19. Loria, A. \& Pan, T. The cleavage step of ribonuclease $P$ catalysis is determined by ribozyme-substrate interactions both distal and proximal to the cleavage site. Biochemistry 38, 8612-8620 (1999).

20. Marvin, M. C. \& Engelke, D. R. Broadening the mission of an RNA enzyme. J. Cell. Biochem. 108, 1244-1251 (2009).

21. Lai, L. B. et al. Discovery of a minimal form of RNase P in Pyrobaculum. Proc. Natl Acad. Sci. USA 107, 22493-22498 (2010).

22. Andrews Andrew, J., Hall Thomas, A. \& Brown James, W. Characterization of RNase P Holoenzymes from Methanococcus jannaschii and Methanothermobacter thermoautotrophicus. Biol. Chem. 382, 1171-1177 (2001)

23. Harris, J. K., Haas, E. S., Williams, D., Frank, D. N. \& Brown, J. W. New insight into RNase P RNA structure from comparative analysis of the archaeal RNA. RNA 7, 220-232 (2001).
24. Tsai, H. Y., Pulukkunat, D. K., Woznick, W. K. \& Gopalan, V. Functional reconstitution and characterization of Pyrococcus furiosus RNase P. Proc. Natl Acad. Sci. USA 103, 16147-16152 (2006).

25. Pannucci, J. A., Haas, E. S., Hall, T. A., Harris, J. K. \& Brown, J. W. RNase P RNAs from some Archaea are catalytically active. Proc. Natl Acad. Sci. USA 96, 7803-7808 (1999)

26. Kouzuma, Y. et al. Reconstitution of archaeal ribonuclease P from RNA and four protein components. Biochem. Biophys. Res. Commun. 306, 666-673 (2003).

27. Terada, A., Honda, T., Fukuhara, H., Hada, K. \& Kimura, M. Characterization of the archaeal ribonuclease P proteins from Pyrococcus horikoshii OT3. J. Biochem. 140, 293-298 (2006).

28. Chen, W. Y., Pulukkunat, D. K., Cho, I. M., Tsai, H. Y. \& Gopalan, V. Dissecting functional cooperation among protein subunits in archaeal RNase P, a catalytic ribonucleoprotein complex. Nucleic Acids Res. 38, 8316-8327 (2010).

29. Fukuhara, H. et al. A fifth protein subunit Ph1496p elevates the optimum temperature for the ribonuclease $\mathrm{P}$ activity from Pyrococcus horikoshii OT3. Biochem. Biophys. Res. Commun. 343, 956-964 (2006).

30. Cho, I. M., Lai, L. B., Susanti, D., Mukhopadhyay, B. \& Gopalan, V. Ribosomal protein L7Ae is a subunit of archaeal RNase P. Proc. Natl Acad. Sci. USA 107, 14573-14578 (2010).

31. Numata, T., Ishimatsu, I., Kakuta, Y., Tanaka, I. \& Kimura, M. Crystal structure of archaeal ribonuclease $\mathrm{P}$ protein Ph1771p from Pyrococcus horikoshii OT3: an archaeal homolog of eukaryotic ribonuclease P protein Rpp29. RNA 10, 1423-1432 (2004).

32. Takagi, H. et al. Crystal structure of the ribonuclease P protein Ph1877p from hyperthermophilic archaeon Pyrococcus horikoshii OT3. Biochem. Biophys. Res. Commun. 319, 787-794 (2004).

33. Kakuta, Y. et al. Crystal structure of a ribonuclease P protein Ph1601p from Pyrococcus horikoshii OT3: an archaeal homologue of human nuclear ribonuclease P protein Rpp21. Biochemistry 44, 12086-12093 (2005).

34. Kawano, S., Nakashima, T., Kakuta, Y., Tanaka, I. \& Kimura, M. Crystal structure of protein Ph1481p in complex with protein Ph1877p of archaeal RNase P from Pyrococcus horikoshii OT3: implication of dimer formation of the holoenzyme. J. Mol. Biol. 357, 583-591 (2006).

35. Wilson, R. C., Bohlen, C. J., Foster, M. P. \& Bell, C. E. Structure of Pfu Pop5, an archaeal RNase P protein. Proc. Natl Acad. Sci. USA 103, 873-878 (2006).

36. Honda, T., Kakuta, Y., Kimura, K., Saho, J. \& Kimura, M. Structure of an archaeal homolog of the human protein complex Rpp21-Rpp29 that is a key core component for the assembly of active ribonuclease P. J. Mol. Biol. 384, 652-662 (2008).

37. Oshima, K. et al. Structural basis for recognition of a kink-turn motif by an archaeal homologue of human RNase P protein Rpp38. Biochem. Biophys. Res. Commun. 474, 541-546 (2016).

38. Oshima, K. et al. Crystal structures of the archaeal RNase P protein Rpp38 in complex with RNA fragments containing a K-turn motif. Acta Crystallogr. F. Struct. Biol. Commun. 74, 57-64 (2018).

39. Wu, J. et al. Cryo-EM Structure of the Human Ribonuclease P Holoenzyme. Cell 175, 1393-1404 el311 (2018).

40. Lan, P. et al. Structural insight into precursor tRNA processing by yeast ribonuclease P. Science 362, eaat6678 (2018).

41. Pulukkunat, D. K. \& Gopalan, V. Studies on Methanocaldococcus jannaschi RNase P reveal insights into the roles of RNA and protein cofactors in RNase P catalysis. Nucleic Acids Res. 36, 4172-4180 (2008).

42. Gao, X., Oshima, K., Ueda, T., Nakashima, T. \& Kimura, M. A threedimensional model of RNase $\mathrm{P}$ in the hyperthermophilic archaeon Pyrococcus horikoshii OT3. Biochem. Biophys. Res. Commun. 493, 1063-1068 (2017).

43. Lai, S. M., Lai, L. B., Foster, M. P. \& Gopalan, V. The L7Ae protein binds to two kink-turns in the Pyrococcus furiosus RNase P RNA. Nucleic Acids Res. 42 13328-13338 (2014)

44. Lai, L. B. et al. A novel double kink-turn module in euryarchaeal RNase P RNAs. Nucleic Acids Res. 45, 7432-7440 (2017).

45. Hall, T. A. \& Brown, J. W. Interactions between RNase P protein subunits in archaea. Archaea 1, 247-254 (2004)

46. Kifusa, M., Fukuhara, H., Hayashi, T. \& Kimura, M. Protein-Protein Interactions in the Subunits of Ribonuclease P in the Hyperthermophilic Archaeon Pyrococcus horikoshii OT3. Biosci. Biotechnol. Biochem. 69, 1209-1212 (2005)

47. Hartmann, E. \& Hartmann, R. K. The enigma of ribonuclease P evolution. Trends Genet. 19, 561-569 (2003).

48. Yuan, Y. \& Altman, S. Substrate recognition by human RNase P: identification of small, model substrates for the enzyme. EMBO J. 14, 159-168 (1995).

49. Liu, F. \& Altman, S. Requirements for cleavage by a modified RNase P of a small model substrate. Nucleic Acids Res. 24, 2690-2696 (1996).

50. Atassi, M. Z., Liu, F. \& Altman, S. Protein Reviews: Ribonuclease P, 1 edn (Springer-Verlag, New York, 2010). 
51. Sinapah, S. et al. Cleavage of model substrates by archaeal RNase P: role of protein cofactors in cleavage-site selection. Nucleic Acids Res. 39, 1105-1116 (2011).

52. Kimura, M. Structural basis for activation of an archaeal ribonuclease $P$ RNA by protein cofactors. Biosci. Biotechnol. Biochem. 81, 1670-1680 (2017).

53. Haas, E. S. \& Brown, J. W. Evolutionary variation in bacterial RNase P RNAs. Nucleic Acids Res. 26, 4093-4099 (1998).

54. Daniels, C. J., Armbruster, D. W., Vucson, B. M., Haas, E. S. \& Brown, J. W. Comparative Analysis of Ribonuclease P RNA Structure in Archaea. Nucleic Acids Res. 24, 1252-1259 (1996).

55. Kirsebom, L. A. \& Trobro, S. RNase P RNA-mediated cleavage. IUBMB Life 61, 189-200 (2009).

56. Perreault, J. P. \& Altman, S. Important 2'-hydroxyl groups in model substrates for M1 RNA, the catalytic RNA subunit of RNase P from Escherichia coli. J. Mol. Biol. 226, 399-409 (1992).

57. Fedor, M. J. The role of metal ions in RNA catalysis. Curr. Opin. Struct. Biol. 12, 289-295 (2002).

58. Liu, X., Chen, Y. \& Fierke, C. A. Inner-sphere coordination of divalent metal ion with nucleobase in catalytic RNA. J. Am. Chem. Soc. 139, 17457-17463 (2017).

59. Steitz, T. A. \& Steitz, J. A. A general two-metal-ion mechanism for catalytic RNA. Proc. Natl Acad. Sci. USA 90, 6498-6502 (1993).

60. Hsieh, J. et al. A divalent cation stabilizes the active conformation of the $\mathrm{B}$. subtilis RNase P x pre-tRNA complex: a role for an inner-sphere metal ion in RNase P. J. Mol. Biol. 400, 38-51 (2010).

61. Christian, E. L., Smith, K. M., Perera, N. \& Harris, M. E. The P4 metal binding site in RNase P RNA affects active site metal affinity through substrate positioning. RNA 12, 1463-1467 (2006).

62. Beebe, J. A., Kurz, J. C. \& Fierke, C. A. Magnesium ions are required by bacillus subtilis ribonuclease P RNA for both binding and cleaving precursor tRNAAsp. Biochemistry 35, 10493-10505 (1996).

63. Scott, W. G. \& Klug, A. Ribozymes: structure and mechanism in RNA catalysis. Trends Biochem. Sci. 21, 220-224 (1996).

64. Brown, J. W. et al. Comparative analysis of ribonuclease P RNA using gene sequences from natural microbial populations reveals tertiary structura elements. Proc. Natl Acad. Sci. USA 93, 3001-3006 (1996).

65. Mastronarde, D. N. Automated electron microscope tomography using robust prediction of specimen movements. J. Struct. Biol. 152, 36-51 (2005).

66. Tang, G. et al. EMAN2: An extensible image processing suite for electron microscopy. J. Struct. Biol. 157, 38-46 (2007)

67. Scheres, S. H. Processing of Structurally Heterogeneous Cryo-EM Data in RELION. Methods Enzymol. 579, 125-157 (2016).

68. Zheng, S. Q. et al. MotionCor2: anisotropic correction of beam-induced motion for improved cryo-electron microscopy. Nat. Methods 14, 331-332 (2017).

69. Zhang, K. Gctf: Real-time CTF determination and correction. J. Struct. Biol. 193, 1-12 (2016)

70. Demeshkina, N., Jenner, L., Westhof, E., Yusupov, M. \& Yusupova, G. A new understanding of the decoding principle on the ribosome. Nature 484, 256-259 (2012).
71. Hamma, T. \& Ferré-D’Amaré, A. R. Structure of protein L7Ae bound to a Kturn derived from an archaeal box H/ACA sRNA at $1.8 \AA$ resolution. Structure 12, 893-903 (2004).

\section{Acknowledgements}

We thank the staff members of the Electron Microscopy System, specially, M. Cao, for his help on data collection. And we also thank the Mass Spectrometry System at Shanghai Institute of Precision Medicine for providing technical support and assistance in data collection. This work was supported by grants from the National Natural Science Foundation of China (31525007 to M.L.), the Strategic Priority Research Program of the Chinese Academy of Sciences (XDB08010201 to M.L.) and the Young Elite Scientist Sponsorship Program of China Association for Science and Technology (2018QNRC001 to P.L.).

\section{Author contributions}

F.W., J.T., J.C., and S.S. reconstituted the MjaRNase P complex. Q.W. and M.T. prepared cryo-EM specimens, collected datasets and determined the structures. J.W. carried out model building and refinement. All the authors were involved in data interpretation and contributed the writing of the manuscript. M.L., Q.W., and P.L. wrote the manuscript. M.L., J.W., and P.L. initiated and orchestrated the project.

\section{Additional information}

Supplementary Information accompanies this paper at https://doi.org/10.1038/s41467019-10496-3.

Competing interests: The authors declare no competing interests.

Reprints and permission information is available online at http://npg.nature.com/ reprintsandpermissions/

Journal Peer Review Information Nature Communications thanks the anonymous reviewer(s) for their contribution to the peer review of this work. Peer reviewer reports are available.

Publisher's note: Springer Nature remains neutral with regard to jurisdictional claims in published maps and institutional affiliations.

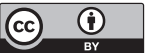

Open Access This article is licensed under a Creative Commons Attribution 4.0 International License, which permits use, sharing, adaptation, distribution and reproduction in any medium or format, as long as you give appropriate credit to the original author(s) and the source, provide a link to the Creative Commons license, and indicate if changes were made. The images or other third party material in this article are included in the article's Creative Commons license, unles indicated otherwise in a credit line to the material. If material is not included in the article's Creative Commons license and your intended use is not permitted by statutory regulation or exceeds the permitted use, you will need to obtain permission directly from the copyright holder. To view a copy of this license, visit http://creativecommons.org/ licenses/by/4.0/

(c) The Author(s) 2019 